\title{
An Adaptive Direction-Dependent Polarization State Configuration Method for High Isolation in Polarimetric Phased Array Radar
}

This paper was downloaded from TechRxiv (https://www.techrxiv.org).

\section{LICENSE}

CC BY 4.0

SUBMISSION DATE / POSTED DATE

04-11-2020 / 16-11-2020

\section{CITATION}

Wang, Zhanling; Yin, Jiapeng; Pang, Chen; Li, Yongzhen; Wang, Xuesong (2020): An Adaptive DirectionDependent Polarization State Configuration Method for High Isolation in Polarimetric Phased Array Radar. TechRxiv. Preprint. https://doi.org/10.36227/techrxiv.13186769.v1

\section{DOI}




\title{
An Adaptive Direction-Dependent Polarization State Configuration Method for High Isolation in Polarimetric Phased Array Radar
}

\author{
Zhanling Wang, Jiapeng Yin, Chen Pang, Yongzhen Li, and Xuesong Wang
}

\begin{abstract}
High cross-polarization isolation (CPI) is crucial to the accurate polarization measurement using polarimetric phased array radar (PPAR). In this paper, we propose an adaptive direction-dependent polarization state configuration ( $\left.\mathrm{AD}^{2} \mathrm{PSC}\right)$ method to improve the polarization isolation. Compared with conventional fixed polarization state of radiated wave whether it is linear, circular or elliptical polarization state, our $\mathrm{AD}^{2} \mathrm{PSC}$ approach configures the polarization state on basis of beam steering. To achieve the adaptive configuration of magnitude and phase of the dual-polarization antenna, an improved steepest descent algorithm is put forward. To facilitate the uniform representation for the polarization measurement application of PPAR, the universal expressions of intrinsic and measured backscatter matrices are derived for arbitrary polarization state. The dualpolarization dipole array is used to assess the priority of our proposed method. Compared with the conventional approaches, our approach could obtain higher CPI while being available for a larger scanning range. The configured CPI meets the specific polarization requirement for PPAR.
\end{abstract}

Index Terms-Cross-polarization isolation, elliptical polarization, polarimetric phased array radar antenna, polarization state configuration.

\section{INTRODUCTION}

$\mathbf{R}$ ECENTLY, polarimetric phased array radar (PPAR) has attracted widespread attention due to the capability of electronic scanning and agile beam, which is advantageous for weather observation, air surveillance, and air traffic control [1]-[7]. However, there are some challenges. For example, the electric fields emanating from the vertical (V) and horizontal $(\mathrm{H})$ ports are not necessarily orthogonal when the beam is pointed off broadside [3]. This results in a production of the cross-polar component and the cross-polarization isolation (CPI) deteriorates appreciably, thereby generating the polarization measurement bias. The existing problems cannot meet the high accuracy requirement for polarimetric measurement, thereby hindering the development of PPAR [7]-[9]. Therefore, the potential of PPAR could be realized only if the bias caused by the cross-polar component is mitigated considerably. Aiming at this problem, some researchers have proposed the calibration/correction methods which are summarized as follows.

Manuscript received XX xx, 2020; revised XX xx, 2020. This work is supported by the National Natural Science Foundation of China (NSFC) under Grant No. 61701512, No. 61625108, and No. 61971429. (Corresponding author: Zhanling Wang)

The authors are with State Key Laboratory of Complex Electromagnetic Environment Effects on Electronics and Information System, National University of Defense Technology, Changsha 410073, China (e-mail: zlwangnudt@outlook.com).
Signal processing. Bias correction to the received echo is implemented in signal processing. Zhang et al. firstly put forward [3] the correction technique for polarization information measurement in PPAR. The projection matrix method in [3] has been developed in [9]-[12], and further updated by Fulton et al. [13], and demonstrated on the large-scale testbeds in [5], [14]. Specifically, a multiplication operation is performed on the measured polarization scattering matrix (PSM) using the correction matrix. So far, most of the bias corrections focus on the narrow-band case. An method, which is the expansion of projection matrix correction method in [3], is presented for the wideband PPAR [15]. In addition, because of the inevitable distortion of radiation pattern mainly resulting from the mutual coupling [16], the correction matrix using the theoretical pattern is not completely effective. To mitigate the effect of non-ideality on the polarization measurement, a pattern reconstruction method is put forward in [17], [18].

Antenna design. Currently, many literatures have been reported in the dual-polarization antennas design, mainly from Zhang's team [19]-[25]. The existing antennas for polarization application can be categorized into three separate groups: similar orthogonal elements, a patch antenna having two orthogonal modes, and a dual-polarized antenna with two different radiation elements [26]. The motivation is to improve the polarization isolation and polarization purity of dualpolarization antennas. However, the polarization state is fixed for the radiated electric field once the antenna design is completed. Moreover, the cross-polar level will increase with the deviation angle away from the broadside, especially for a planar PPAR (PPPAR). Hence, the PPPAR has inherent limitation in realizing accurate polarimetric measurement due to its changing pattern characteristics with the beam scanning. The cylindrical PPAR (CPPAR) is one of the most promising concepts for eliminating the cross-polar of the PPPAR, with the radiated beam always staying broadside to the antenna plane [14], [27].

Beamforming. The cross-polarization pattern could be suppressed by using the beamforming algorithm [28]. A spacetime-polarization filter structure is proposed for the pattern synthesis of the conformal phased array, and the alternating projection method is used to depress the cross-polar level in [29]. Then, the antenna pattern synthesis is considered with polarization constraints using an array of vector antennas [30]. A number of intelligent optimization algorithms provide an opportunity for digital beamforming (DBF), thereby decreasing the cross-polarization level and sidelobes. A hybrid particle 
swarm optimization-gravitational search algorithm (PSOGSA) is investigated to control each element optimally for the pattern synthesis in [31]. And the performance of the proposed method are illustrated using a practical experiment [32]. Moreover, a semidefinite relaxation method is presented for the linearly polarized pattern shaping, thereby reducing the crosspolarization and sidelobe levels [33]. However, the optimum operations need to be realized for each element in the specified beam direction. Moreover, the computation complexity will be problematic for real-time requirement.

Phase coding. Phase coding between pulses is the technique that can reduce the impact of cross-polarization. It has been proposed in [34], evaluated in [35], experimentally tested in [36] and summarized in [37]. The phase coding method can mitigate the differential reflectivity bias and reduce the differential phase and copolar correlation coefficient bias. The first-order crosspolar term is removed effectively, thereby reducing the crosspolarization in the Alternate Transmission and Simultaneous Reception (ATSR) mode. However, the second-order terms with respect to cross-polar, do not get removed in this phase coding technique.

The above-mentioned calibration/correction methods are helpful in suppressing the polarization measurement bias from a direct or indirect perspective. However, the source of bias remains in the Signal processing method. The cross-polarization cannot be reduced too much in the Antenna design method. The high complexity of the advanced optimization algorithms hinders the practicality of the Beamforming method. In addition, the second-order terms of the cross-polar cannot be removed in the Phase coding method. Furthermore, the bias increases with the gain of the beam steering angle away from the principle plane, which applies to all the calibration/correction methods. If the CPI can be designed as large as possible within the whole beam scanning angle, the accuracy of the polarization measurement could be greatly improved, which is the motivation of this paper.

Within this context, the polarization state configuration (PSC) technique is a candidate scheme to improve CPI. The polarization state can be represented by a prominent geometric representation of polarization known as the Poincare sphere [38], [39]. And each polarization state could be denoted by a point on the surface of a unit sphere. The relative amplitude and phase characteristics of two orthogonal electric field vectors could be mapped to the sphere's surface. In actually, the electronically scanned beam of PPAR will be steered to many spatial directions within a wide steering range. For a given beam direction, the lower the crosspolarization, the better. Thus, the polarization state should be modulated by appropriately varying the magnitude ratio and phase difference of the dual-polarization antenna. Since the cross-polar isolation requirement of the accurate polarimetric measurement is $-22 \mathrm{~dB}$ for the ATSR mode, which is stricter for the Simultaneous Transmission and Simultaneous Reception (STSR) mode (-40 dB) [14], [40]. Hence, it is necessary to modulate the polarization state optimally to reduce the cross-polar level. In this paper, we focus on addressing the cross-polarization isolation. To simplify our analysis, the noise increase or the error or precision of the excitation voltage in practice are ignored or idealized.

In this article, we present an novel adaptive directiondependent polarization state configuration ( $\mathrm{AD}^{2} \mathrm{PSC}$ ) method to solve the aforementioned problems. The configuration operation is dependent on the beam pointing, further finding the optimal polarization state adaptively. Therefore, the problem is described as a minimum problem with specific parameter constraints. Through modulating the magnitude and phase of two polarized ports according to beam direction, the polarization state of the radiated wave is not the linearly polarized wave but the elliptically polarized wave, including the right-hand elliptically polarized (RHEP) and left-hand elliptically polarized (LHEP). If we let the LHEP component be the co-polarization, the RHEP component will be the crosspolarization, and vice versa.

Numerical simulations verify the feasibility of the proposed method. From the comparison of CPI and pattern synthesis, the configured elliptical polarization (C-EP) possesses the apparent superiority with respect to the conventional non-configured polarization states which are referred to as non-configured linear polarization (NC-LP) and non-configured circular polarization (NC-CP) in this work. Furthermore, the $\mathrm{AD}^{2}$ PSC method has favorable effect on reducing the biases of the polarimetric variables.

The article is organized as follows. Section II presents the formulation of the $\mathrm{AD}^{2} \mathrm{PSC}$ method based on a basic element. To optimize the calculation of configuration parameters, the improved steepest descent algorithm is put forword. In Section III, the application on the polarization measurement in PPAR is presented. The array pattern is synthesized, and the scattering matrix and the polarimetric variables are formulated. The results and analysis for a basic element are elaborated in Section IV including the spatial polarization characteristics, performance of $\mathrm{AD}^{2} \mathrm{PSC}$ method, and the parameter sensitivity discussion. The performance of the proposed method is analyzed based on the polarimetric phased array in Section $\mathrm{V}$. The priority of $\mathrm{C}$-EP is verified relative to NC-LP and NC-CP. Section VI describes the conclusions and discussions.

\section{THE $\mathrm{AD}^{2}$ PSC METHOD}

In this section, the $\mathrm{AD}^{2} \mathrm{PSC}$ method is formulated based on a basic element. A formula is deduced using the radiation characteristics of the antenna theoretically. We choose the coordinate system and the dual-polarization element is located at the origin. As displayed in Fig.1(a), the antenna plane is in the yoz plane. We derive the isolation model of the single element using the general elliptically polarized wave. Then, the steepest descent algorithm is improved to facilitate the configuration of polarization state adaptively.

\section{A. Formulation based on a Basic Element}

As shown in Fig.1(a), a pair of crossed dipoles is composed of port 1 and port 2. When these two ports are activated separately, the radiated electric field vectors can be expressed as

$$
\begin{aligned}
\mathbf{E}_{q} & =E_{q} \mathbf{e}_{q} \\
& =E_{q \theta} \mathbf{a}_{\theta}+E_{q \phi} \mathbf{a}_{\phi}
\end{aligned}
$$




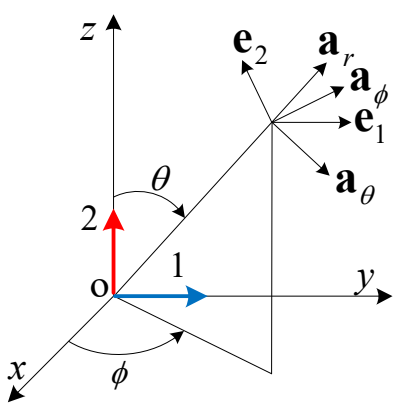

(a)

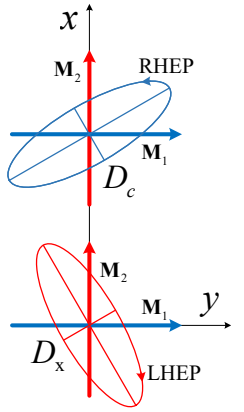

(b)
Fig. 1. A basic element. (a) Spherical coordinate system for crossed dipoles. (b) A basic element for transmission and reception.

where $q$ denotes the $q$ th port, $q=1$ or $2 . \mathbf{e}_{q}, \mathbf{a}_{\phi}$ and $\mathbf{a}_{\theta}$ are unit vectors and their definitions are consistent with that in [3], [11], [41]. $\mathbf{E}_{q}$ is the vector form of $E_{q}$.

As shown in Fig.1(b), we assume that the upper crossed dipoles $D_{c}$ could radiate the RHEP wave and lower crossed dipoles $D_{\mathrm{x}}$ could radiate the LHEP wave. For the $D_{c}$ or $D_{\mathrm{x}}$ antenna, the elliptical polarization is obtained by the superposition of the electric fields from the horizontally and vertically polarized dipoles, i.e., port 1 and port 2 . The radaited electric fields of port 1 and port 2 in $D_{c}$ are defined as $\mathbf{E}_{c 1}$ and $\mathbf{E}_{c 2}$, respectively. The magnitude ratio of electric fields is given by $p_{c}=\left|\mathbf{E}_{c 2}\right| /\left|\mathbf{E}_{c 1}\right|$. Moreover, the excitation phases of port 1 and port 2 are defined as $\varphi_{c 1}$ and $\varphi_{c 2}$, separately. The phase difference is defined as $\varphi_{c}=\varphi_{c 2}-\varphi_{c 1}$ and $-\pi<\varphi_{c}<0$.

Similarly, the radaited electric fields of two ports in $D_{\mathrm{x}}$ are given by $\mathbf{E}_{\mathrm{x} 1}$ and $\mathbf{E}_{\mathrm{x} 2}$, respectively, and the corresponding magnitude ratio is $p_{\mathrm{x}}=\left|\mathbf{E}_{\mathrm{x} 2}\right| /\left|\mathbf{E}_{\mathrm{x} 1}\right|$. The excitation phases of the crossed dipoles of $D_{\mathrm{x}}$ are $\varphi_{\mathrm{x} 1}$ and $\varphi_{\mathrm{x} 2}$, separately. The phase difference is $\varphi_{\mathrm{x}}=\varphi_{\mathrm{x} 2}-\varphi_{\mathrm{x} 1}$ where $0<\varphi_{\mathrm{x}}<\pi$.

Therefore, the RHEP and LHEP waves radiated from the $D_{c}$ and $D_{\mathrm{x}}$ can be given by

$$
\left\{\begin{array}{l}
\mathbf{E}_{\tilde{R}}=\left[\begin{array}{ll}
1 & e^{\mathrm{j} \varphi_{c}}
\end{array}\right]\left[\begin{array}{l}
\mathbf{E}_{c 1} \\
\mathbf{E}_{c 2}
\end{array}\right] \\
\mathbf{E}_{\tilde{L}}=\left[\begin{array}{ll}
1 & e^{\mathrm{j} \varphi_{\mathrm{x}}}
\end{array}\right]\left[\begin{array}{l}
\mathbf{E}_{\mathrm{x} 1} \\
\mathbf{E}_{\mathrm{x} 2}
\end{array}\right]
\end{array}\right.
$$

To ensure the opposite rotation direction of $\mathbf{E}_{\tilde{R}}$ and $\mathbf{E}_{\tilde{L}}$, the excitation should be alternate. That is, port 1 of $D_{\mathrm{x}}$ is excited by $\varphi_{c 2}$ and port 2 by $\varphi_{c 1}$. Thus, $\varphi_{\mathrm{x} 1}=\varphi_{c 2}, \varphi_{\mathrm{x} 2}=\varphi_{c 1}$, and $\varphi_{\mathrm{x}}=-\varphi_{c}$. Without loss of generality, $\varphi_{c}$ is abbreviated as $\varphi$ below. Without considering the mutual coupling between antennas $D_{c}$ and $D_{\mathrm{x}}$, the horizontally polarized dipoles in $D_{c}$ and $D_{\mathrm{x}}$ should have the same radiation characteristics, i.e., $\mathbf{E}_{c 1}=\mathbf{E}_{\mathrm{x} 1}$. The same is true for the vertically polarized dipoles, i.e., $\mathbf{E}_{c 2}=\mathbf{E}_{\mathrm{x} 2}$. Then, $p_{\mathrm{x}}=p_{c}$ and $p_{c}$ is abbreviated as $p$ below. The magnitude ratio of electric fields is proportional to that of excitation voltages.

In addition, according to formula (1), formula (2) can be rewritten as

$$
\begin{aligned}
{\left[\begin{array}{l}
\mathbf{E}_{\tilde{R}} \\
\mathbf{E}_{\tilde{L}}
\end{array}\right] } & =\left[\begin{array}{cc}
1 & e^{\mathrm{j} \varphi} \\
1 & e^{-\mathrm{j} \varphi}
\end{array}\right]\left[\begin{array}{l}
\mathbf{E}_{1} \\
\mathbf{E}_{2}
\end{array}\right] \\
& =\left[\begin{array}{cc}
E_{1 \phi}+e^{\mathrm{j} \varphi} E_{2 \phi} & E_{1 \theta}+e^{\mathrm{j} \varphi} E_{2 \theta} \\
E_{1 \phi}+e^{-\mathrm{j} \varphi} E_{2 \phi} & E_{1 \theta}+e^{-\mathrm{j} \varphi} E_{2 \theta}
\end{array}\right]\left[\begin{array}{l}
\mathbf{a}_{\phi} \\
\mathbf{a}_{\theta}
\end{array}\right]
\end{aligned}
$$

The unit vectors of $\mathbf{E}_{\tilde{\mathbf{R}}}$ and $\mathbf{E}_{\tilde{\mathbf{L}}}$ are expressed as $\mathbf{e}_{\tilde{\mathbf{R}}}$ and $\mathbf{e}_{\tilde{\mathbf{L}}}$, respectively. This pair of unit vectors is written as

$$
\left[\begin{array}{c}
\mathbf{e}_{\tilde{R}} \\
\mathbf{e}_{\tilde{L}}
\end{array}\right]=\frac{E_{1}}{E_{\tilde{R}}}\left[\begin{array}{cc}
\bar{E}_{1 \phi}+e^{\mathrm{j} \varphi} p \bar{E}_{2 \phi} & \bar{E}_{1 \theta}+e^{\mathrm{j} \varphi} p \bar{E}_{2 \theta} \\
\bar{E}_{1 \phi}+e^{-\mathrm{j} \varphi} p \bar{E}_{2 \phi} & \bar{E}_{1 \theta}+e^{-\mathrm{j} \varphi} p \bar{E}_{2 \theta}
\end{array}\right]\left[\begin{array}{c}
\mathbf{a}_{\phi} \\
\mathbf{a}_{\theta}
\end{array}\right]
$$

where the bar on variable $E$ indicates the normalized electric field component of a horizontally/vertically dipole. $\bar{E}_{q t}=$ $E_{q t} / E_{q}$ where $q=1,2$ and $t=\phi, \theta$.

Ideally, the polarization states of antennas $D_{c}$ and $D_{\mathrm{x}}$ should be orthogonal to each other, thereby having the superior crosspolarization isolation. According to formula (4), $\mathbf{e}_{\tilde{\mathbf{R}}}$ and $\mathbf{e}_{\tilde{\mathbf{L}}}$ are not orthonomal and cannot form the orthonormal basis. However, the motivation of the proposed $\mathrm{AD}^{2} \mathrm{PSC}$ method is to enhance the orthonormality of $\mathbf{e}_{\tilde{\mathbf{R}}}$ and $\mathbf{e}_{\tilde{\mathbf{L}}}$ for the specific beam direction. It is worth noting that even if $\mathbf{e}_{\tilde{\mathbf{R}}}$ and $\mathbf{e}_{\tilde{\mathbf{L}}}$ are orthogonal, the orthogonality cannot be maintained because of the transmission medium or the radiating system. The corresponding orthogonality can be restored by using a differential phase shifter and a differential attenuator [42]. Therefore, we refer to $\left\{\mathbf{e}_{\tilde{R}}, \mathbf{e}_{\tilde{L}}\right\}$ as the elliptical polarization "basis" herein.

If the activated crossed dipoles product elliptically polarized wave, and the radiated electric field is projected onto the elliptically polarized "basis", then

$$
E_{x y}=\left\langle\mathbf{e}_{x}, \mathbf{E}_{y}\right\rangle
$$

where $x, y=\tilde{R}$ or $\tilde{L} . E_{x y}(x=y)$ and $E_{x y}(x \neq y)$ are the copolar and cross-polar electric fields, respectively. By simplifying and omitting the common coefficient, the expansion is given by

$$
\left\{\begin{array}{c}
E_{\tilde{R} \tilde{R}}=\bar{E}_{1 \phi}^{2}+2(\cos \varphi) p \bar{E}_{1 \phi} \bar{E}_{2 \phi}+p^{2} \bar{E}_{2 \phi}^{2}+ \\
\bar{E}_{1 \theta}^{2}+2(\cos \varphi) p \bar{E}_{1 \theta} \bar{E}_{2 \theta}+p^{2} \bar{E}_{2 \theta}^{2} \\
E_{\tilde{R} \tilde{L}}=\left(\bar{E}_{1 \phi}+e^{-\mathrm{j} \varphi} p \bar{E}_{2 \phi}\right)^{2}+\left(\bar{E}_{1 \theta}+e^{-\mathrm{j} \varphi} p \bar{E}_{2 \theta}\right)^{2} \\
E_{\tilde{L} \tilde{L}}=\bar{E}_{1 \phi}^{2}+2(\cos \varphi) p \bar{E}_{1 \phi} \bar{E}_{2 \phi}+p^{2} \bar{E}_{2 \phi}^{2}+ \\
\bar{E}_{1 \theta}^{2}+2(\cos \varphi) p \bar{E}_{1 \theta} \bar{E}_{2 \theta}+p^{2} \bar{E}_{2 \theta}^{2} \\
E_{\tilde{L} \tilde{R}}=\left(\bar{E}_{1 \phi}+e^{\mathrm{j} \varphi} p \bar{E}_{2 \phi}\right)^{2}+\left(\bar{E}_{1 \theta}+e^{\mathrm{j} \varphi} p \bar{E}_{2 \theta}\right)^{2}
\end{array}\right.
$$

To keep the rotation direction of the elliptical polarization wave unchanged, let $-\pi \leq \varphi \leq 0$ herein. Moreover, the ratio of excitation magnitude is limited within $\pm 3 \mathrm{~dB}$, that is, $0.5 \leq p \leq 2$.

CPI denotes the relation between copolar and cross-polar power on the decibel scale. The bigger the value of CPI, the greater the isolation between copolar and cross-polar electric fields. When setting the RHEP wave as the co-polarization, the CPI is given by

$$
\mathrm{CPI}_{\tilde{R}}=20 \log \left(\frac{E_{\tilde{R} \tilde{R}}}{E_{\tilde{R} \tilde{L}}}\right)
$$


For simplicity, let $\mathrm{CPI}_{\tilde{R}}=20 \log (1 / \rho)$ where

$$
\begin{aligned}
\rho & =\frac{E_{\tilde{R} \tilde{L}}}{E_{\tilde{R} \tilde{R}}} \\
& =\frac{\bar{E}_{1 \phi}^{2}+2 e^{-j \varphi} p \bar{E}_{1 \phi} \bar{E}_{2 \phi}+e^{-2 j \varphi} p^{2} \bar{E}_{2 \phi}^{2}+\bar{E}_{1 \theta}^{2}+2 e^{-j \varphi} p \bar{E}_{1 \theta} \bar{E}_{2 \theta}+e^{-2 j \varphi} p^{2} \bar{E}_{2 \theta}^{2}}{\bar{E}_{1 \phi}^{2}+2(\cos \varphi) p \bar{E}_{1 \phi} \bar{E}_{2 \phi}+p^{2} \bar{E}_{2 \phi}^{2}+\bar{E}_{1 \theta}^{2}+2(\cos \varphi) p \bar{E}_{1 \theta} \bar{E}_{2 \theta}+p^{2} \bar{E}_{2 \theta}^{2}}
\end{aligned}
$$

In equation (8), $\rho$ represents the ratio of copolar and crosspolar electric fields, it is equivalent to equation (7). Enhancing the $\mathrm{CPI}_{\tilde{R}}$ could be transformed into the reducing of $\rho$. Since the co- and cross-polarization components are functions of $(\varphi, p)$, CPI raising problem can be modeled as an optimization problem. Being subject to a set of constraint conditions, the objective function is given by

$$
\begin{array}{ll}
\min _{\varphi, p} & \rho(\varphi, p) \\
\text { s.t. } & \left\{\begin{array}{c}
-\pi \leq \varphi \leq 0 \\
0.5 \leq p \leq 2
\end{array}\right.
\end{array}
$$

For a known beam direction $(\phi, \theta)$, the minimum problem is to find the optimal phase difference $\varphi$ and amplitude ratio $p$, thereby reducing $\rho$. Each pair of parameters calculated corresponds to an individual elliptical polarization state. $\varphi$ and $p$ jointly determine the ellipticity angle, orientation angle, and rotation direction of the polarized ellipse. Hence, the polarization state configuration is implemented based on the minimum problem.

However, the characteristics of the objective function depend on the beam direction, so the configuration operation is also direction-dependent. The differentiability, monotonicity, and convexity of the objective function should be considered. To solve this problem, we propose the improved steepest descent algorithm to conduct the configuration operation adaptively in Part B of this Section. The proposed method that configures the direction-dependent polarization state adaptively is named as the $\mathrm{AD}^{2} \mathrm{PSC}$ method, and it offers a scheme to raise the CPI considerably and keep the co-polarization acceptably.

The flowchart of the $\mathrm{AD}^{2} \mathrm{PSC}$ method is shown in Fig. 2. For a given beam direction or an estimated direction of arrival, $(\phi, \theta)$, the electric field vector of the antenna could be determined. When the activated $D_{c}$ products RHEP wave, the copolar and cross-polar electric fields in the single direction could be expressed by substituting $(\phi, \theta)$ into $E_{\tilde{R} \tilde{R}}$ and $E_{\tilde{R} \tilde{L}}$, respectively. Then, the objective function $\rho(\varphi, p)$ corresponding to $(\phi, \theta)$ is determined where $-\pi \leq \varphi \leq 0$ and $0.5 \leq p \leq 2$. By calculating the minimum of the function, the corresponding phase difference $\varphi$ and amplitude ratio $p$ are obtained. However, the characteristics of the objective function are highly dependent on the objective direction. If the objective function is not differentiable at the extreme points, the conventional minimum solution method represented by the steepest descent algorithm is not feasible. In this case, the minimum cannot be found. Thus, an improved steepest descent algorithm in this paper to solve the problem and the detailed analysis are described in Part B of this Section below. A pair of polarization parameters $\varphi$ and $p$ is obtained once the minimum $\rho_{\min }$ is found. Finally, two polarized-ports of $D_{c}$ shown in Fig.1(b) are activated by the calculated complex voltage. The polarization state configuration for the superimposed radiated

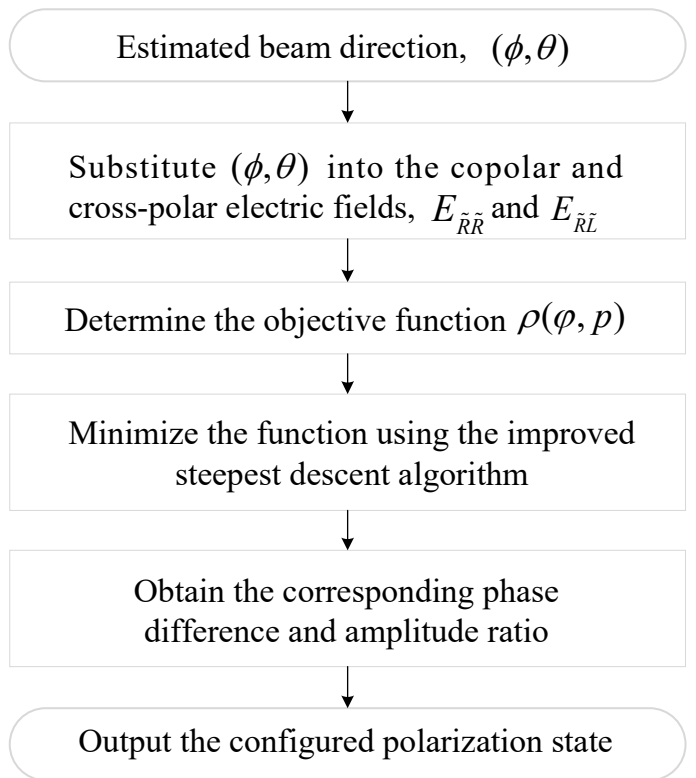

Fig. 2. The flowchart of the proposed $\mathrm{AD}^{2} \mathrm{PSC}$ method.

electric field vectors is achieved. For each direction, the $\mathrm{AD}^{2} \mathrm{PSC}$ method is implemented accordingly.

The $\mathrm{AD}^{2} \mathrm{PSC}$ method can adjust the phased difference $\varphi$ and amplitude ratio $p$ according to different pointing directions, which means that the polarization state is adaptively configured. In this case, most of the polarized waves are elliptical polarization which is referred to as C-EP in this paper. However, if the $\varphi$ and $p$ are all consistent, the polarization state is fixed. For example, the NC-LP and NC-CP are two special cases of the non-configured polarization states, which are widely used nowadays. Specifically, NC-LP is utilized in the polarimetric weather radar, while $\mathrm{NC}-\mathrm{CP}$ in the spaceborne radar and mobile communication. The performance comparison between the NC-LP, NC-CP, and C-EP is presented next in the theoretical derivation and application on the polarization measurement.

\section{B. Improved Steepest Descent Algorithm}

An improved steepest descent algorithm is presented on basis of the crossed dipoles antenna. The algorithm aims to determine the optimal polarization parameters, thereby configuring the polarization state adaptively. As shown in Fig.1(b), each element of PPAR antenna is composed of two pairs of crossed dipoles which are placed orthogonally. $D_{c}$ and $D_{\mathrm{x}}$ compose a transceiver module. $D_{c}$ not only radiates modulated polarized wave but also receives the scattered wave, while $D_{\mathrm{x}}$ does not radiate but receives the scattered wave. The amplitude ratio of the upper crossed dipoles is $p$ and that of the lower ones is $p$ as well. The phase difference of the upper crossed dipoles is $\varphi$, but that of the lower ones is opposite, $-\varphi$. The co-polarization component is obtained by $D_{c}$ and the cross-polarization component by $D_{\mathrm{x}}$ when the dual-polarization antenna is used.

To complete the separation of co- and cross-polarization components, four dipoles are needed for the polarization 
configuration operation. On the one hand, the configured elliptically polarized antenna is essentially the combination of two linearly polarized dipoles. On the other hand, the cross-polarization component is obtained by another configured elliptically polarized antenna which is also composed of two linearly polarized dipoles.

The dipole moment is given by

$$
\mathbf{M}_{q}=\mathbf{a}_{q} A_{q} e^{j \varphi_{q}}
$$

where $A_{q}$ and $\varphi_{q}$ are excitation amplitude and phase respectively. And the unit vector $\mathbf{a}_{q}$ is along the dipole direction. $q$ (1 or 2 ) denotes the $q$ th port.

The radiated electric field is

$$
\mathbf{E}_{q}=-\frac{k^{2} e^{-\mathrm{j} k r}}{4 \pi \varepsilon r}\left\{\mathbf{a}_{r} \times\left[\mathbf{a}_{r} \times \mathbf{M}_{q}\right]\right\}
$$

where $r$ is the observation distance, $k$ the wavenumber, and $k=2 \pi / \lambda, \lambda$ the wavelength, $\varepsilon$ the permittivity.

Based on (10)-(11), the radiated electric field vectors of port 1 and 2 are

$$
\left\{\begin{array}{l}
\mathbf{E}_{1}=\left(\mathbf{a}_{\theta} \cos \theta \sin \phi+\mathbf{a}_{\phi} \cos \phi\right) E_{1} \\
\mathbf{E}_{2}=-\mathbf{a}_{\theta}(\sin \theta) E_{2}=-\mathbf{a}_{\theta}(\sin \theta) p E_{1}
\end{array}\right.
$$

Formula (3) is rewritten as

$$
\left[\begin{array}{l}
\mathbf{E}_{\tilde{R}} \\
\mathbf{E}_{\tilde{L}}
\end{array}\right]=E_{1}\left[\begin{array}{cc}
\cos \phi & \cos \theta \sin \phi-p e^{\mathrm{j} \varphi} \sin \theta \\
\cos \phi & \cos \theta \sin \phi-p e^{-\mathrm{j} \varphi} \sin \theta
\end{array}\right]\left[\begin{array}{l}
\mathbf{a}_{\phi} \\
\mathbf{a}_{\theta}
\end{array}\right]
$$

where the spatial domain is constrained within $0 \leq \theta \leq \pi$, $-\pi / 2 \leq \phi \leq \pi / 2$.

Based on (6), when the activated crossed dipoles $D_{c}$ products RHEP wave, the spatial copolar and cross-polar electric fields are

$\left\{E_{\tilde{R} \tilde{R}}=E_{1}^{2}\left(\cos ^{2} \phi+\cos ^{2} \theta \sin ^{2} \phi-p \cos \varphi \sin \phi \sin 2 \theta+p^{2} \sin ^{2} \theta\right)\right.$

$\left\{E_{\tilde{R} \tilde{L}}=E_{1}^{2}\left(\cos ^{2} \phi+\cos ^{2} \theta \sin ^{2} \phi-e^{-\mathrm{j} \varphi} p \sin \phi \sin 2 \theta+e^{-2 \mathrm{j} \varphi} p^{2} \sin ^{2} \theta\right)\right.$

Similarly, when the LHEP wave is radiated, the spatial co-polarization $E_{\tilde{L} \tilde{L}}$ and cross-polarization $E_{\tilde{L} \tilde{R}}$ components could be deduced.

Therefore, formula (8) is rewritten as

$\rho=\frac{\cos ^{2} \phi+\cos ^{2} \theta \sin ^{2} \phi-e^{-\mathrm{j} \varphi} p \sin \phi \sin 2 \theta+e^{-2 \mathrm{j} \varphi} p^{2} \sin ^{2} \theta}{\cos ^{2} \phi+\cos ^{2} \theta \sin ^{2} \phi-p \cos \varphi \sin \phi \sin 2 \theta+p^{2} \sin ^{2} \theta}$

For simplicity, we can set $A=\cos \theta \sin \phi, B=\cos \phi$, $C=\sin \theta$. The optimal polarization state is configured for a given beam direction $(\phi, \theta)$. To be specific, the corresponding polarization parameters $(\varphi, p)$ are calculated for the current beam direction, thereby minimizing the electric field ratio $\rho$. The expression is given by

$$
\begin{aligned}
\rho_{\min } & =\min _{\substack{-\pi \leq \varphi \leq 0 \\
0.5 \leq p \leq 2}} \rho(\theta, \phi) \\
& =\min _{\substack{-\pi \leq \varphi \leq 0 \\
0.5 \leq p \leq 2}} \frac{B^{2}+A^{2}-2 e^{-\mathrm{j} \varphi} p A C+e^{-2 \mathrm{j} \varphi} p^{2} C^{2}}{B^{2}+A^{2}-2(\cos \varphi) p A C+p^{2} C^{2}}
\end{aligned}
$$

The minimum of $\rho$ is not explicit, so we try to deduce it based on its partial derivative. For the multivariable function, the partial derivative of $\rho$ with respect to $\varphi$ is

$$
\begin{aligned}
\frac{\partial \rho}{\partial \varphi}= & \frac{\mathrm{j} 2 e^{-\mathrm{j} \varphi} p A C-\mathrm{j} 2 e^{-2 \mathrm{j} \varphi} p^{2} C^{2}}{A^{2}+B^{2}-2 p A C \cos \varphi+p^{2} C^{2}}- \\
& \frac{2 p A C \sin \varphi\left(A^{2}+B^{2}-2 e^{-\mathrm{j} \varphi} p A C+e^{-2 \mathrm{j} \varphi} p^{2} C^{2}\right)}{\left(A^{2}+B^{2}-2 p A C \cos \varphi+p^{2} C^{2}\right)^{2}}
\end{aligned}
$$

Similarly, the partial derivative of $\rho$ concerning $p$ is

$$
\begin{aligned}
\frac{\partial \rho}{\partial p}= & \frac{-2 e^{-\mathrm{j} \varphi} A C+2 e^{-2 \mathrm{j} \varphi} p C^{2}}{A^{2}+B^{2}-2 p A C \cos \varphi+p^{2} C^{2}}- \\
& \frac{\left(A^{2}+B^{2}-2 e^{-\mathrm{j} \varphi} p A C+e^{-2 \mathrm{j} \varphi} p^{2} C^{2}\right)\left(-2 A C \cos \varphi+2 p C^{2}\right)}{\left(A^{2}+B^{2}-2 p A C \cos \varphi+p^{2} C^{2}\right)^{2}}
\end{aligned}
$$

We assume that the numerator of the partial derivative is zero, that is $\left\{\frac{\partial \rho}{\partial \varphi}, \frac{\partial \rho}{\partial p}\right\}=\{0,0\}$. After discarding the complex solution, the real solution is

$$
\left\{\begin{array}{l}
\varphi=-\pi \text { or } 0 \\
p=0
\end{array}\right.
$$

The partial derivative is deduced analytically, but the partial derivative could be zero only in the minimum or maximum of $\varphi$ value. Otherwise, the $\left\{\frac{\partial \rho}{\partial \varphi}, \frac{\partial \rho}{\partial p}\right\}$ is not equal to $\{0,0\}$. Therefore, the objective function $\rho$ is not necessarily differentiable in the extreme point.

The minimum of objective function cannot be computed analytically, so an iterative method to obtain an approximate solution could be used. As we know, the steepest descent method is an effective method for finding the nearest local minimum of a function which presupposes that the gradient of the function can be calculated. Accordingly, if the method of steepest descent is used, we firstly let an initial point $\mathbf{x}_{0}=\left[\varphi_{0}, p_{0}\right]^{T}$, and the direction of steepest descent is the vector $-\nabla \rho\left(\mathbf{x}_{0}\right)$ where $\nabla \rho\left(\mathbf{x}_{0}\right)$ is the local downhill gradient $\left[\frac{\partial \rho}{\partial \varphi_{0}}, \frac{\partial \rho}{\partial p_{0}}\right]^{T}$. The method takes the form of iterating $\rho\left(\mathbf{x}_{\kappa+1}\right)=\rho\left(\mathbf{x}_{\kappa}+l_{\kappa} \nabla \rho\left(\mathbf{x}_{\kappa}\right)\right)$ through the iteration times $\kappa$, where $l_{\kappa}$ is the stepsize. The iteration operation is stopped until $\left\|\nabla \rho\left(\mathbf{x}_{\kappa}\right)\right\| \leq \varepsilon$ is reached.

However, the gradient $\left\|\nabla \rho\left(\mathbf{x}_{\kappa}\right)\right\|$ may not have good convergence due to that $\rho$ is not necessarily differentiable at the extreme point as derived above. The iteration process does not converge and the minimum of the objective function $\rho$ cannot be found. Therefore, the conventional steepest descent method cannot be utilized directly. It needs to be improved especially on the convergence condition.

In this situation, we modify the conventional method and propose the improved steepest descent method. The iteration process is determined by the objective function itself but not its downhill gradient. The framework of the modified steepest descent method is displayed in Algorithm.1. The stepsize $l_{\kappa}$ is modulated across with the iteration times $\kappa$. Herein, a one-dimensional optimization method is used in updating the stepsize. 


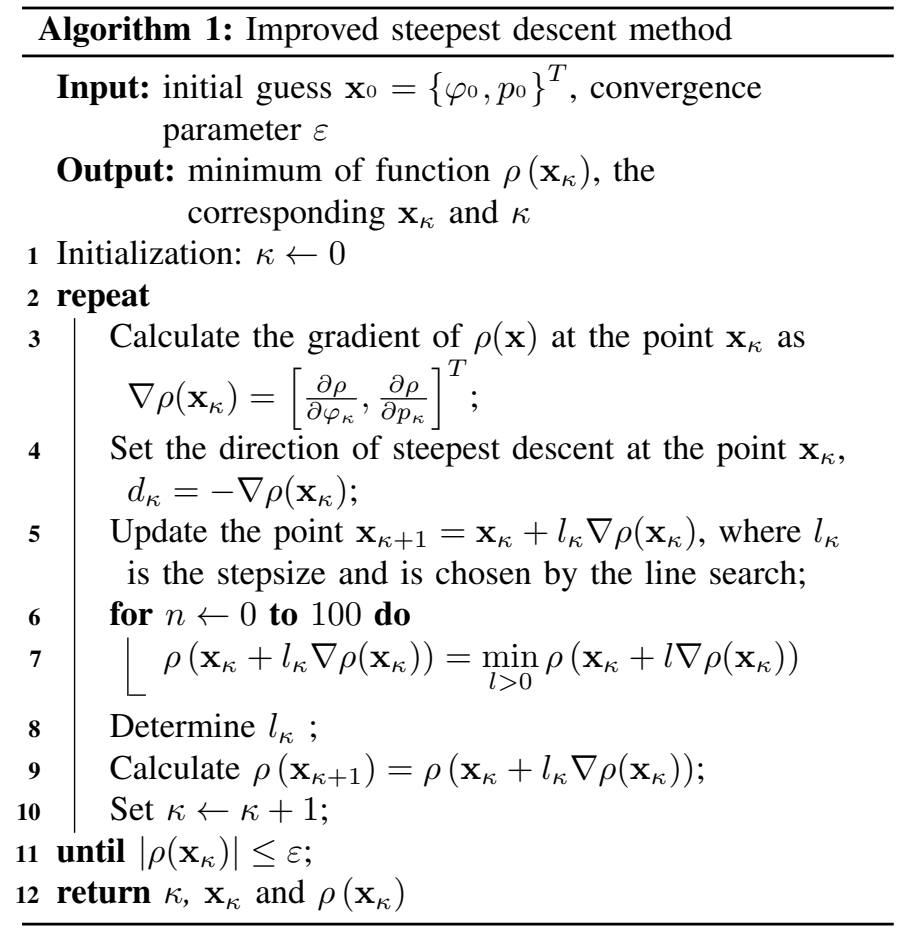

\section{Application on the Polarization Measurement IN THE POLARIMETRIC PHASED ARRAY}

In this section, the application of the $\mathrm{AD}^{2} \mathrm{PSC}$ method on the pattern synthesis and polarization measurement in the polarimetric phased array is presented. Firstly, the array pattern is synthesized for the constructed phased array architecture based on the $\mathrm{AD}^{2} \mathrm{PSC}$ method. Secondly, the universal polarization scattering matrix is derived based on the arbitrary polarized wave. Finally, the accuracy of the polarization measurement is assessed by the polarimetric variables.

\section{A. Pattern Synthesis}

Each antenna element is composed of two dual-polarization crossed dipoles, as displayed in Fig.1(b). The upper one is used to radiate the polarized wave and receive the copolar electric field. The lower one is utilized to only receive the cross-polarization component.

For a uniformly spaced linear array with $N$ elements, we assume that the element spacing is half-wavelength. The radiated electric field can be given by the superposition of element pattern, so the array pattern of the horizontally/vertically polarized ports array is given by

$$
\mathbf{F}_{q}(\phi, \theta)=\sum_{n=1}^{N} w_{n} \mathbf{E}_{q_{n}}(\phi, \theta)
$$

where $q$ indicates the $q$ th port of $D_{c}$ or $D_{\mathrm{x}} . \mathbf{F}_{q}(\phi, \theta)$ represents the array pattern vector radiated from all the $q$ ports of $N$ elements. For the $n$th element, $\mathbf{E}_{q n}(\phi, \theta)$ is the corresponding element pattern vector and $w_{n}$ is the complex weight coefficient. In addition to the directionality of the patterns, we also investigate the polarimetric characteristics.

We assume that the mutual coupling between the elements is ignored herein. Hence, the complete array pattern can be found using pattern multiplication theorem which states that the complete array pattern can be calculated by multiplying the array factor and element pattern. Thus, (21) is rewritten as

$$
\mathbf{F}_{q}(\phi, \theta)=\mathbf{E}_{q}(\phi, \theta) \sum_{n=1}^{N} w_{n}
$$

If the radiated polarized wave is configured into the RHEP state, the combined array pattern according to the deduction above is

$$
\begin{aligned}
\mathbf{F}_{\tilde{R}}(\phi, \theta) & =\mathbf{F}_{1}(\phi, \theta)+e^{\mathrm{j} \varphi} \mathbf{F}_{2}(\phi, \theta) \\
& =\mathbf{E}_{\tilde{R}}(\phi, \theta) \sum_{n=1}^{N} w_{n}
\end{aligned}
$$

For simplicity, $\mathbf{F}_{\tilde{R}}(\phi, \theta)$ and $\mathbf{E}_{\tilde{R}}(\phi, \theta)$ are expressed as $\mathbf{F}_{\tilde{R}}$ and $\mathbf{E}_{\tilde{R}}$ below, respectively. The unit vector of $\mathbf{F}_{\tilde{R}}$ is consistent with that of $\mathbf{E}_{\tilde{R}}$. Project $\mathbf{F}_{\tilde{R}}$ onto the elliptical polarization vector $\mathbf{e}_{\tilde{R}}$, the co- and cross-polarization components of the electric fields are expressed as

$$
\left\{\begin{array}{l}
F_{\tilde{R} \tilde{R}}=\left\langle\mathbf{e}_{n \tilde{R}}, \mathbf{F}_{\tilde{R}}\right\rangle=E_{n \tilde{R} \tilde{R}} \sum_{n=1}^{N} w_{n} \\
F_{\tilde{R} \tilde{L}}=\left\langle\mathbf{e}_{n \tilde{R}}, \mathbf{F}_{\tilde{L}}\right\rangle=E_{n \tilde{R} \tilde{L}} \sum_{n=1}^{N} w_{n}
\end{array}\right.
$$

Similarly, if the configured polarization state is LHEP, the cross-polarization component $F_{\tilde{L} \tilde{R}}$ and co-polarization component $F_{\tilde{L} \tilde{L}}$ using the complete array pattern could be obtained.

A polarimetric phased array architecture using the conventional linearly polarized wave non-configured is shown in Fig. 3(a). The horizontally and vertically dipoles are activated by the source signal modulated by the transmit/reveive (T/R) module. The array architecture could radiate and receive the strictly $\mathrm{H} / \mathrm{V}$ polarized wave. The scattered profile is separated into two parts: copolar and cross-polar electric fields. If this array architecture is used for the polarization measurement, the measurement bias will be introduced and the corresponding correction to bias is needed. The bias increases with the gain of steering angle, thereby limiting the beam scanning performance of the polarimetric phased array [3]. Similarly, the problem cannot be avoided for the array using the non-configured circularly polarized wave. Furthermore, another important flaw of the circularly polarized wave is that the coherency matrix measured by circular polarization basis can not be well connected with the bulk precipitation properties for the weather services [43].

Within this context, the $\mathrm{AD}^{2} \mathrm{PSC}$ method is proposed, and it can be achieved conveniently in different phased array architectures. Take the digital phased array for example, a block diagram of the adjusted architecture is displayed in Fig. 3(b). In the array, the generated signals for each element are configured and then up-converted into radio frequency signals. The signals are radiated from each element and scattered from the observation object. The scattered wave illuminates the array aperture and is received by $N$ elements. After the configuring of amplitude and phase in $\mathrm{T} / \mathrm{R}$ modules and beamforming, digital signal processing is done for various special-purpose radar applications. 


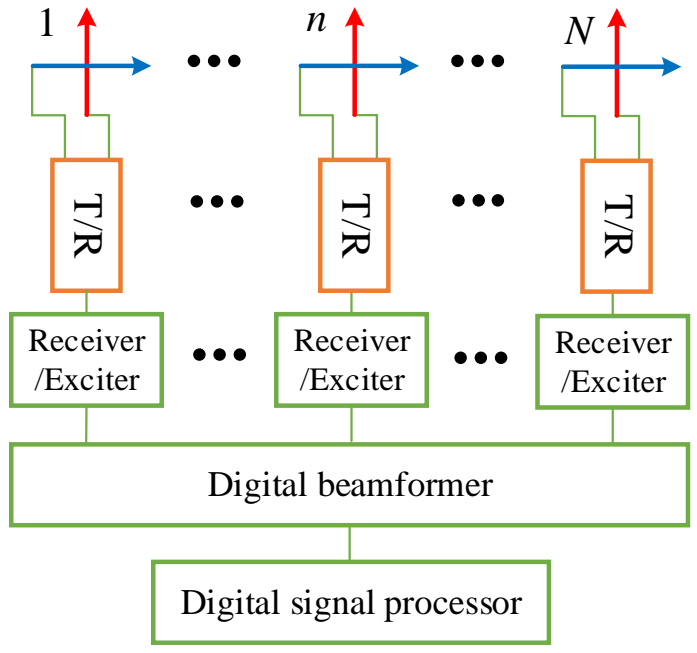

(a)

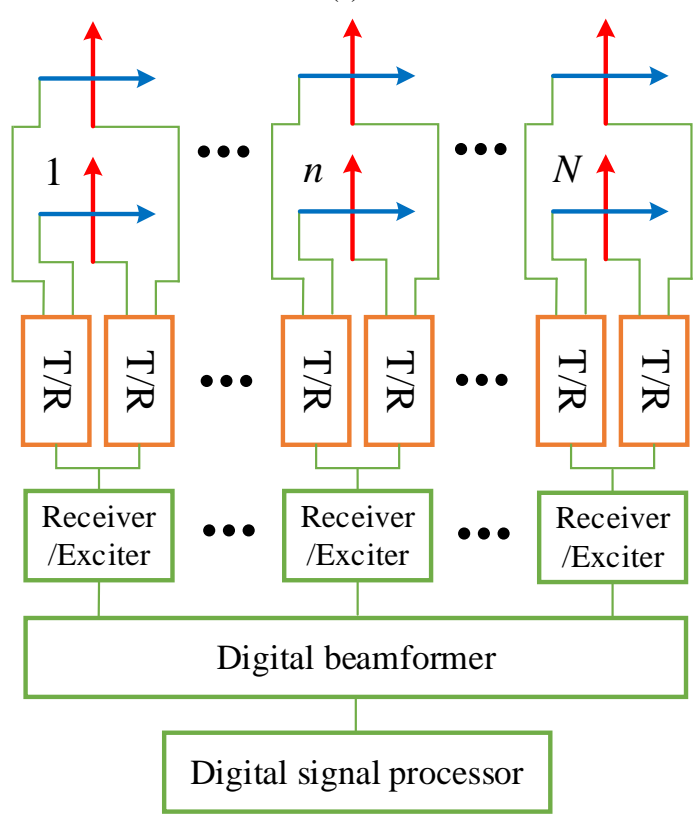

(b)

Fig. 3. The digital phased array architecture. (a) The architecture using the conventional linearly polarized wave. (b) The architecture applying the $\mathrm{AD}^{2} \mathrm{PSC}$ method.

The adjusted architecture is the expansion of conventional digital phased array architecture from element-level to portlevel. Our polarization configuration method makes full use of the resources of the T/R module, thereby extending the spatial domain scanning to the mixture of spatial domain and polarization domain scanning. Specifically, to form the desired beam, the signal of each element needs to be configured in amplitude and phase using T/R modules. The outputs of the $\mathrm{AD}^{2} \mathrm{PSC}$ method in this paper are also amplitude and phase. Two types of configurations can be synthesized and implemented together in T/R modules, without effecting the beam scanning. For each row, the modulation in an array composed of horizontally or vertically polarized ports is just related to spatial domain scanning. However, the modulation between horizontally ports and vertically ports is different and direction-dependent, and this is the polarization domain manipulation. The first row of crossed dipoles array is used for transmitting and receiving, and the corresponding modulation is done twice. The second row is used just for receiving, with the corresponding modulation on the received signals in $T / R$ modules.

Consider the practical realization, the steps for polarization measurement are as follows:

- Step 1: According to the direction of arrival of the observed object, $(\phi, \theta)$, calculate the phase difference $\varphi$ and amplitude ratio $p$ using the proposed $\mathrm{AD}^{2} \mathrm{PSC}$ method including the improved steepest descent algorithm.

- Step 2: Configure the polarization states of two-row array based on $(\varphi, p)$, thereby obtaining a set of polarization states such as RHEP and LHEP, which are as orthogonal as possible.

- Step 3: Get the copolar and cross-polar electric fields of the scattered wave, thereby obtaining the scattering matrix representing the object characteristics.

\section{B. Polarization Scattering Matrix}

We can ultilize the ATSR or STSR modes to measure the components of PSM using the polarimetric antenna alternately or simultaneously. For the ATSR mode, all four components of PSM could be estimated using two or more pulses. After radiating, scattering, and propagating of the electromagnetic signal, the measured PSM can be expressed as

$$
\mathbf{S}^{(m)}=\mathbf{P}^{T} \mathbf{S}^{(i)} \mathbf{P}
$$

where $\mathbf{S}^{(i)}$ is the intrinsic (i) PSM of the target, while $\mathbf{S}^{(m)}$ is the measured $(m)$ PSM. $\mathbf{P}$ denotes the projection matrix representing the projections of the radiation electric fields on the defined polarization ' ' basis'. These denotations are consistent with the published literatures about radar polarimetry in [3] [11]. And $T$ means the transpose operator. The observed object is assumed to be a metallic sphere herein.

For the comparison of NC-LP, NC-CP, and CEP waves, the corresponding scattering matrices are denoted by $\mathbf{S}_{L P}^{(\eta)}=\left[S_{\phi \phi}^{(\eta)}, S_{\phi \theta}^{(\eta)} ; S_{\theta \phi}^{(\eta)}, S_{\theta \theta}^{(\eta)}\right]_{2 \times 2}$, $\mathbf{S}_{C P}^{(\eta)}=\left[S_{R R}^{(\eta)}, S_{R L}^{(\eta)} ; S_{L R}^{(\eta)}, S_{L L}^{(\eta)}\right]_{2 \times 2}, \quad \mathbf{S}_{E P}^{(\eta)}=$ $\left[S_{\tilde{R} \tilde{R}}^{(\eta)}, S_{\tilde{R} \tilde{L}}^{(\eta)} ; S_{\tilde{L} \tilde{R}}^{(\eta)}, S_{\tilde{L} \tilde{L}}^{(\eta)}\right]_{2 \times 2}$ respectively, where $\eta=i$ or $m$.

For the NC-LP wave, $\stackrel{\mathbf{P}}{L P}^{2}$ denotes the projection matrix on the linear polarization basis $\left\{\mathbf{a}_{\phi}, \mathbf{a}_{\theta}\right\}$, and $\mathbf{S}_{L P}^{(i)}$ is considered to be a unit matrix herein. Hence, the measured scattering matrix is written as

$$
\begin{aligned}
\mathbf{S}_{L P}^{(m)} & =\left[\begin{array}{cc}
f_{\phi \phi} & f_{\phi \theta} \\
f_{\theta \phi} & f_{\theta \theta}
\end{array}\right]^{T}\left[\begin{array}{ll}
1 & 0 \\
0 & 1
\end{array}\right]\left[\begin{array}{cc}
f_{\phi \phi} & f_{\phi \theta} \\
f_{\theta \phi} & f_{\theta \theta}
\end{array}\right] \\
& =\left[\begin{array}{cc}
f_{\phi \phi}^{2}+f_{\theta \phi}^{2} & f_{\phi \phi} f_{\phi \theta}+f_{\theta \phi} f_{\theta \theta} \\
f_{\phi \phi} f_{\phi \theta}+f_{\theta \phi} f_{\theta \theta} & f_{\theta \theta}^{2}+f_{\phi \theta}^{2}
\end{array}\right]
\end{aligned}
$$

For the NC-CP wave, $\mathbf{P}_{C P}$ is the projection matrix on the circular polarization basis $\left\{\mathbf{e}_{R}, \mathbf{e}_{L}\right\}$ where $\mathbf{e}_{R}=\frac{1}{\sqrt{2}}\left(\mathbf{a}_{\phi}+\mathbf{j} \mathbf{a}_{\theta}\right)$ 
and $\mathbf{e}_{L}=\frac{1}{\sqrt{2}}\left(\mathbf{a}_{\phi}-\mathbf{j} \mathbf{a}_{\theta}\right)$. According to polarization transformation theory in [44], [45], the transformation matrix $\mathbf{U}(R L \rightarrow \phi \theta)$ (from NC-CP to NC-LP) is defined as

$$
\mathbf{U}(R L \rightarrow \phi \theta)=\frac{1}{\sqrt{2}}\left[\begin{array}{cc}
1 & 1 \\
-\mathrm{j} & \mathrm{j}
\end{array}\right] .
$$

Thus,

$$
\begin{aligned}
\mathbf{S}_{C P}^{(i)} & =[\mathbf{U}(R L \rightarrow \phi \theta)]^{T} \mathbf{S}_{L P}^{(i)} \mathbf{U}(R L \rightarrow \phi \theta) \\
& =\left[\begin{array}{ll}
0 & 1 \\
1 & 0
\end{array}\right]
\end{aligned}
$$

Hence, if a right-hand circularly polarized (RHCP) wave is incident on the scatterer $\left(E_{R}=1, E_{L}=0\right)$, the back scattered wave is left-hand circularly polarized (LHCP), i.e., the received polarization sense is opposite to the transmitted sense. Therefore, the measured PSM is given by

$$
\begin{aligned}
\mathbf{S}_{C P}^{(m)} & =\left[\begin{array}{ll}
f_{R R} & f_{R L} \\
f_{L R} & f_{L L}
\end{array}\right]^{T}\left[\begin{array}{ll}
0 & 1 \\
1 & 0
\end{array}\right]\left[\begin{array}{cc}
f_{R R} & f_{R L} \\
f_{L R} & f_{L L}
\end{array}\right] \\
& =\left[\begin{array}{cc}
2 f_{R R} f_{L R} & f_{R R} f_{L L}+f_{L R} f_{R L} \\
f_{R R} f_{L L}+f_{L R} f_{R L} & 2 f_{L L} f_{R L}
\end{array}\right]
\end{aligned}
$$

For the C-EP wave, $\mathbf{P}_{E P}$ describes the projection matrix on the elliptical polarization "basis" $\left\{\mathbf{e}_{\tilde{R}}, \mathbf{e}_{\tilde{L}}\right\}, \mathbf{P}_{E P}=$ $\left[\begin{array}{ll}f_{\tilde{R} \tilde{R}} & f_{\tilde{R} \tilde{L}} \\ f_{\tilde{L} \tilde{R}} & f_{\tilde{L} \tilde{L}}\end{array}\right.$. According to (4), the transformation matrix (from NC-LP to C-EP) is

$$
\mathbf{U}(\phi \theta \rightarrow \tilde{R} \tilde{L})=\frac{E_{1}}{E_{\tilde{R}}}\left[\begin{array}{cc}
\bar{E}_{1 \phi}+e^{\mathrm{j} \varphi} p \bar{E}_{2 \phi} & \bar{E}_{1 \theta}+e^{\mathrm{j} \varphi} p \bar{E}_{2 \theta} \\
\bar{E}_{1 \phi}+e^{-\mathrm{j} \varphi} p \bar{E}_{2 \phi} & \bar{E}_{1 \theta}+e^{-\mathrm{j} \varphi} p \bar{E}_{2 \theta}
\end{array}\right]
$$

The transformation matrix (from C-EP to NC-LP) is the inverse matrix of (30) and expressed as

$$
\begin{aligned}
\mathbf{U}(\tilde{R} \tilde{L} \rightarrow \phi \theta) & =[\mathbf{U}(\phi \theta \rightarrow \tilde{R} \tilde{L})]^{-1} \\
& =\tilde{\mathbf{U}}\left[\begin{array}{cc}
\bar{E}_{1 \theta}+e^{-\mathrm{j} \varphi} p \bar{E}_{2 \theta} & -\bar{E}_{1 \theta}-e^{\mathrm{j} \varphi} p \bar{E}_{2 \theta} \\
-\bar{E}_{1 \phi}-e^{-\mathrm{j} \varphi} p \bar{E}_{2 \phi} & \bar{E}_{1 \phi}+e^{\mathrm{j} \varphi} p \bar{E}_{2 \phi}
\end{array}\right]
\end{aligned}
$$

where $\tilde{\mathbf{U}}=\frac{E_{\tilde{R}}}{\mathrm{j} 2 p E_{1} \sin \varphi\left(\bar{E}_{1 \theta} \bar{E}_{2 \phi}-\bar{E}_{1 \phi} \bar{E}_{2 \theta}\right)}$.

As a special case, $\mathbf{U}(\tilde{R} \tilde{L} \rightarrow \phi \theta)=\mathbf{U}(R L \rightarrow \phi \theta)$ when $\varphi=-\pi / 2$ and $p=1$, i.e., when the elliptically polarized wave is the NC-CP wave.

Thus,

$$
\mathbf{S}_{E P}^{(i)}=[\mathbf{U}(\tilde{R} \tilde{L} \rightarrow \phi \theta)]^{T} \mathbf{S}_{L P}^{(i)} \mathbf{U}(\tilde{R} \tilde{L} \rightarrow \phi \theta)
$$

Therefore, the measured PSM is given by

$$
\mathbf{S}_{E P}^{(m)}=\mathbf{P}_{E P}^{T} \mathbf{S}_{E P}^{(i)} \mathbf{P}_{E P}
$$

The universal expressions of intrinsic and measured backscatter matrices are derived for arbitrary polarization state. The theoretical analysis shows that various polarization states correspond to different scattering matrices for the same scatterer. The deduction provides transformation between the matrices of linearly, circularly and elliptically polarized waves. Thus, the copolar and cross-polar components could be separated in the unified polarization basis.

\section{Polarimetric Variables}

To illustrate the performance of the $\mathrm{AD}^{2} \mathrm{PSC}$ method in polarization measurement application, the differential reflectivity $\left(Z_{D R}\right)$ and linear depolarization ratio $(L D R)$ [44] are utilized. The polarimetric variable $Z_{D R}$ scaled in $\mathrm{dB}$ is given by

$$
Z_{D R(\mathrm{LP} / \mathrm{CP} / \mathrm{EP})}^{(\eta)}=10 \log \left(\frac{\left|S_{\phi \phi / L R / \tilde{L} \tilde{R}}^{(\eta)}\right|^{2}}{\left|S_{\theta \theta / R L / \tilde{R} \tilde{L}}^{(\eta)}\right|^{2}}\right)
$$

where $\eta=i$ or $m$. The subscripts which are separated by slash "p" denote the NC-LP, NC-CP, or C-EP state.

The bias of $Z_{D R}$ is described by $\Delta Z_{D R}$, and the requirement index of $\Delta Z_{D R}$ is less than $0.1 \mathrm{~dB}$ in the accurate polarization measurement application for PPAR [14]

$$
\Delta Z_{D R(\mathrm{LP} / \mathrm{CP} / \mathrm{EP})}=Z_{D R(\mathrm{LP} / \mathrm{CP} / \mathrm{EP})}^{(m)}-Z_{D R(\mathrm{LP} / \mathrm{CP} / \mathrm{EP})}^{(i)}
$$

For the NC-LP wave, the scattering matrix of the metallic sphere is an identity matrix. Thus, $S_{\phi \phi}^{(i)}=S_{\theta \theta}^{(i)}$ and $Z_{D R(\mathrm{LP})}^{(i)}=0$. For the NC-CP wave, the backscattering matrix of the target is an inverse identity matrix, so $S_{L R}^{(i)}=S_{R L}^{(i)}$ and $Z_{D R(\mathrm{CP})}^{(i)}=0$. Hence, for the NC-LP and NC-CP wave, the bias of differential reflectivity is strictly equivalent to the measured value, namely, $\Delta Z_{D R}=Z_{D R(\mathrm{LP} / \mathrm{CP})}^{(m)}$. For the C-EP wave, $\Delta Z_{D R}=Z_{D R(\mathrm{EP})}^{(m)}-Z_{D R(\mathrm{EP})}^{(i)}$.

In addition, the $L D R$ scaled in $\mathrm{dB}$ is expressed as

$$
L D R_{\mathrm{LP}_{\phi} / \mathrm{CP}_{R} / \mathrm{EP}_{\tilde{R}}}^{(\eta)}=10 \log \left(\frac{\left|S_{\theta \phi / R R / \tilde{R} \tilde{R}}^{(\eta)}\right|^{2}}{\left|S_{\phi \phi / L R / \tilde{L} \tilde{R}}^{(\eta)}\right|^{2}}\right)
$$

Consider the assumption that the intrinsic scattering matrices using the NC-LP and NC-CP states are identity matrix and inverse identity matrix, namely, $S_{\phi \theta}^{(i)}=S_{\theta \phi}^{(i)}=0$ and $S_{R R}^{(i)}=$ $S_{L L}^{(i)}=0$, the bias of $L D R$ is equal to the measured $L D R^{(i)}$ Hence, $\Delta L D R=L D R_{\mathrm{LP}_{\phi} / \mathrm{CP}_{R}}^{(m)}$. For C-EP wave, $\Delta L D R=$ $L D R_{\mathrm{EP}_{\tilde{R}}}^{(m)}-L D R_{\mathrm{EP}_{\tilde{R}}}^{(i)}$. Considering the accurate polarization measurement application, the expected requirement of $\Delta L D R$ is constrained less than $-40 \mathrm{~dB}$ herein.

\section{Results AND ANALYSIS FOR A BASIC ELEMENT}

In this section, the comparison is made among the spatial polarization characteristics when radiating the NC-LP, NC$\mathrm{CP}$, and C-EP waves. The performance of $\mathrm{AD}^{2} \mathrm{PSC}$ method is verified on improving the CPI of the radiated electric fields of a basic element antenna. The convergence parameter in the improved steepest descent algorithm is discussed.

\section{A. Spatial Polarization Characteristics}

For the linearly polarized dipole, its pattern of the electric field is given in [3]. The expression of the cross-polar electric field is related to the electromagnetic radiation mechanism. When the radiated polarization state does not match the polarized antenna, the cross-polarization component is produced. As displayed in Fig.4, the spatial polarization characteristics over the whole scanning range are various based on the polarization 


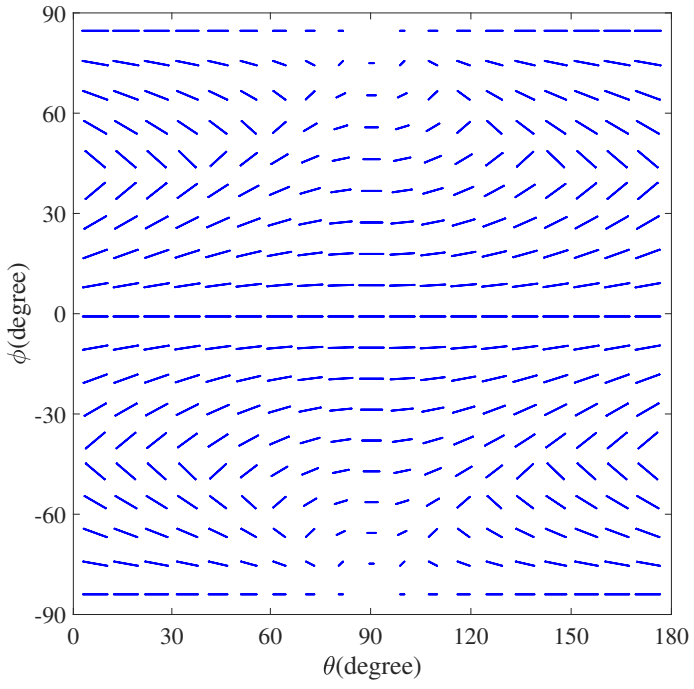

(a)

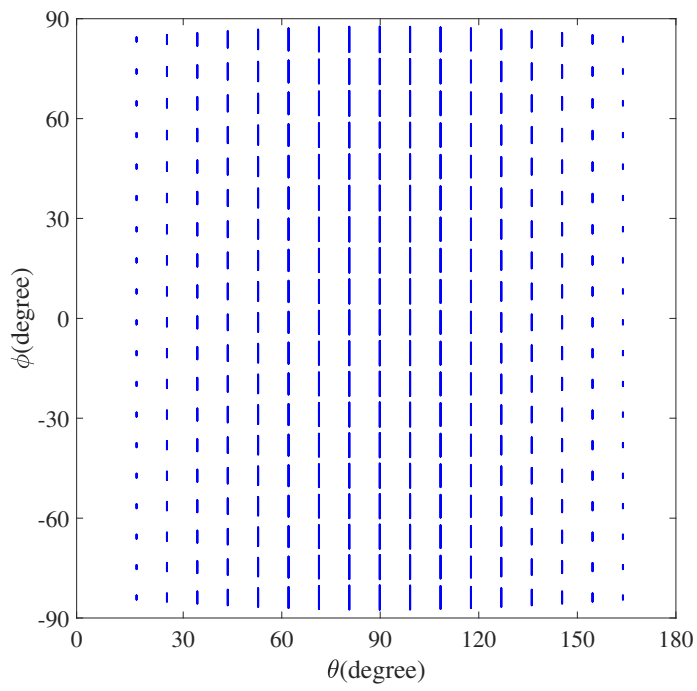

(b)

Fig. 4. Spatial polarization characteristics of dipole with $\phi$ and $\theta$. (a) The horizontally polarized dipole. (b) The vertically polarized dipole. The results show that the polarization state varies usually with the scanning angle.

basis $\left\{\mathbf{a}_{\phi}, \mathbf{a}_{\theta}\right\}$. Fig.4(a) shows that the co-polarization component of the horizontally polarized dipole remains unchanged when $\phi=0^{\circ}$. Thus, the magnitude of the copolar pattern is largest, while the cross-polar pattern is lowest. When the scanning angle is away from the principal plane where $\phi=0^{\circ}$, the polarization state is not horizontal polarization but other linear polarization because of the production of cross-polar electric field. In Fig.4(b), there is only copolar pattern which is increasingly attenuated across with the elevation angle $\theta$, and the polarization state is NC-LP in the overall spatial domain.

We assume that the crossed dipoles radiate circularly polarized wave, and the unit vectors of RHCP and LHCP electric fields are defined as a set of circular polarization basis $\left\{\mathbf{e}_{R}, \mathbf{e}_{L}\right\}$. If the radiated polarization state is LHCP, the spatial polarization characteristics are shown in Fig.5. Most

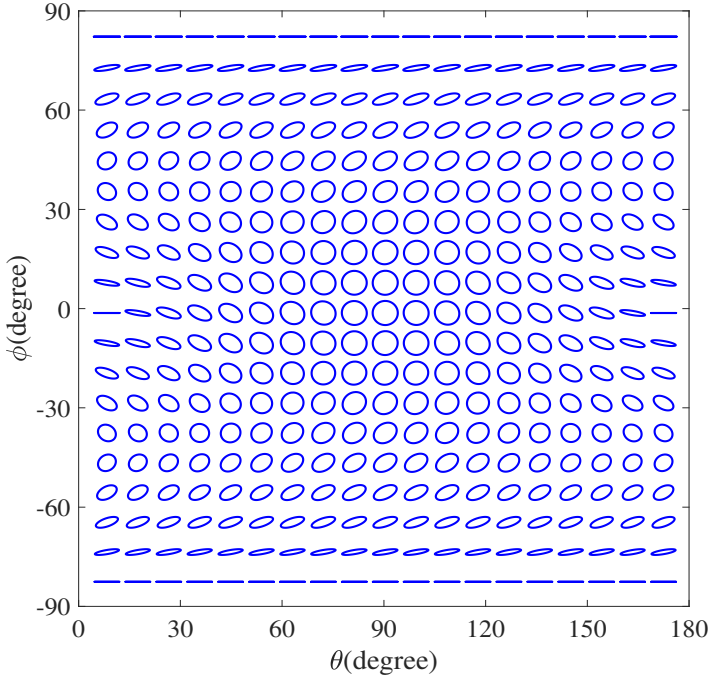

Fig. 5. Spatial polarization characteristics of crossed dipoles with $\phi$ and $\theta$ when radiating LHCP wave. The results reveal that most of the radiated polarization states are not the NC-CP but the elliptical polarization states.

of the polarization states in spatial domain are the elliptical polarization. The original circular polarization state is only distributed around the normal of the antenna plane. Thus, the copolar and cross-polar patterns vary with the azimuth and elevation angles. It can be inferred that the co-polar electric field is the greatest in the normal direction of the antenna plane, that is, $(\phi, \theta)=\left(0^{\circ}, 90^{\circ}\right)$ where the cross-polar electric field is the least. If the beam pointing away from the broadside, the cross-polarization pattern is enhanced and the copolarization is reduced simultaneously. Therefore, the CPI value decreases with the larger angle deviating from the principal plane. The greater the angle, the lower the CPI. The conclusion of the polarization characteristics of the LHCP wave is also suitable for that of the issue when radiating the RHCP wave.

If the amplitude and phase of the upper crossed dipoles $D_{c}$ in Fig.1(b) are modulated, the polarization state of the radiation wave could be configured using the presented $\mathrm{AD}^{2} \mathrm{PSC}$ method. The modulation operation is done for each given direction, and the amplitude and phase are obtained optimally using the improved steepest descent algorithm. If the radiated wave is in LHEP state, the corresponding spatial polarization characteristics of the antenna after configuring are displayed in Fig. 6. Like the polarization characteristics of NC-LP and NC-CP waves, that of the C-EP wave also varies with angles. However, unlike the fixed NC-LP and NC-CP states, the C-EP state is configured adaptively and different from each beam direction. Moreover, the polarization basis of NC-LP or NC$\mathrm{CP}$ state is fixed, while polarization "basis" of C-EP state is various. To illustrate the modulation effect of the radiated polarized field, the CPI index is more suitable for not only the elliptically polarized wave but also the practical performance.

\section{B. Performance of $A D^{2} P S C$ on a Basic Element}

According to the previous discussion, the cross-polarization component increases and the co-polarization component de- 


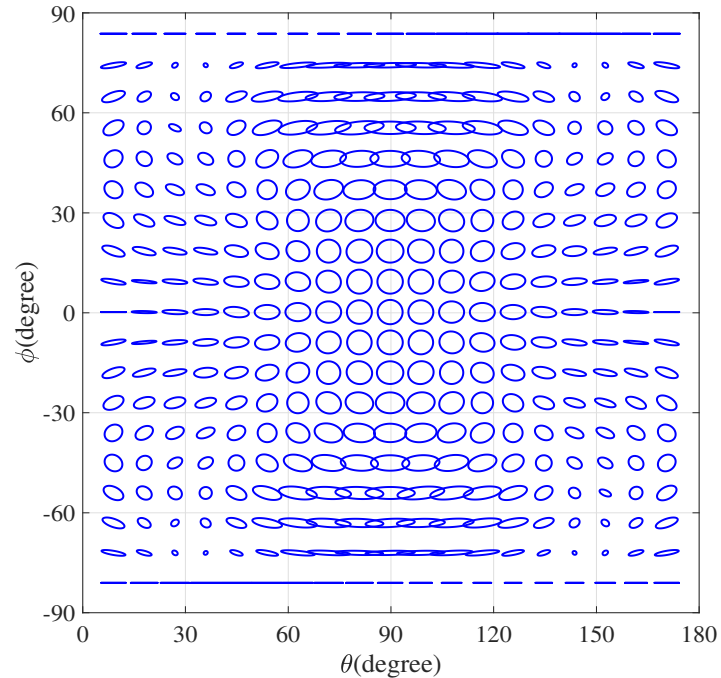

Fig. 6. Spatial polarization characteristics of crossed dipoles with $\phi$ and $\theta$ when radiating the LHEP wave. The results reveal the radiated polarization state which corresponds to each C-EP wave.

creases when the beam direction is pointed off broadside of the horizontally polarized dipole. The beam scanning leads to the reduction of CPI, where the available beam steering range of the antenna is limited. It has been proved that CPI is the key factor for the accurate polarization measurement [46]. The comparison of CPI is shown in Fig. 7 where the sampling interval of angle is $1^{\circ}$. Fig. 7(a) shows the usable angle range when CPI is not less than $40 \mathrm{~dB}$. However, the angle range is sharp and restricted, it is available just around the horizonal and vertical principle planes of the antenna.

The CPI of the NC-CP wave has better symmetry compared with that of the NC-LP wave. The angle range which could meet the requirement (greater than $\geq 40 \mathrm{~dB}$ ) is approximately a circle with a radius $11^{\circ}$ as shown in Fig. 7(b). Although the available range is not broadened compared with that of the NC-LP, it is relatively uniform. Still, NC-CP is not the ideal polarization state that could provide available beam scanning range.

If the polarization state is configured in the expected beam scanning angular sector typically used for a PPAR observation (i.e., $30^{\circ} \leq \theta \leq 150^{\circ},-60^{\circ} \leq \phi \leq 60^{\circ}$ ), the cross-polar level could be suppressed. As shown in Fig. 7(c), the angle range that could meet the CPI requirement is considerably broadened. Compared with the CPI of NC-LP and NC-CP waves as shown in Fig. 7(a)-(b), the CPI of C-EP wave has not only greater value but also a wider considerable angle range, and the advantage is illustrated. To verify the superiority of CEP wave intuitively, the CPI cuts of three types of polarization states over the entire angular sector are displayed in Fig. 8. The black dashed line is the expected CPI requirement $(40 \mathrm{~dB})$. The available elevation angle of C-EP wave spans from $32^{\circ}$ to $148^{\circ}$ if the CPI is required to be greater than $150 \mathrm{~dB}$, and the greatest CPI is $192.5 \mathrm{~dB}$. Similarly, the available azimuth angle spans from $-60^{\circ}$ to $60^{\circ}$ and the greatest CPI is 190.1 $\mathrm{dB}$. Therefore, the CPI of the C-EP wave is far greater than

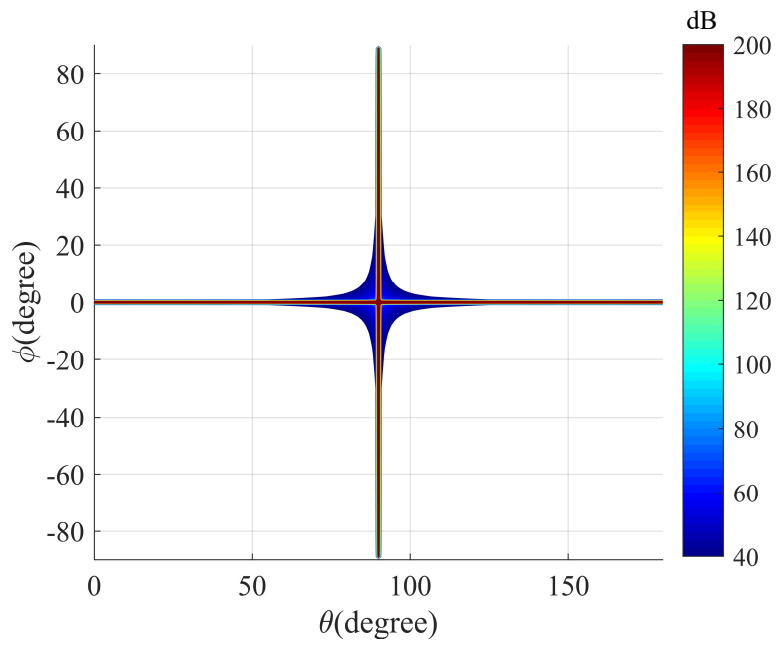

(a)

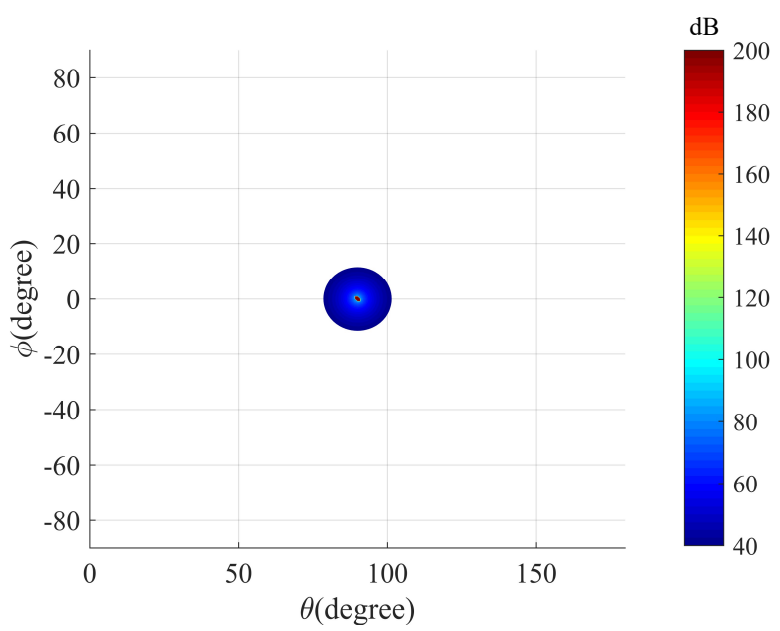

(b)

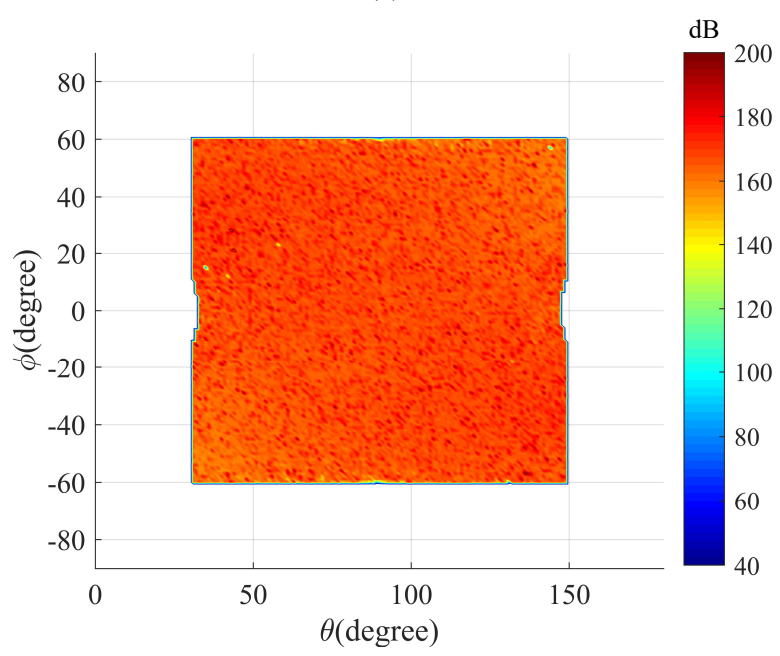

(c)

Fig. 7. The available azimuth and elevation range of different polarized waves when the CPI is not less than $40 \mathrm{~dB}$. (a) NCLP. (b) NC-CP. (c) C-EP. The results reveal that the CPI of C-EP wave has not only greater value $(\geq 150 \mathrm{~dB})$ but also a wider considerable angle range compared with that of NC-LP and $\mathrm{NC}-\mathrm{CP}$ waves. 
the required $40 \mathrm{~dB}$. The available angle range in the whole radiation space is greater than $\pm 58^{\circ}$, which is exactly what the electronically scanned PPAR expects. For the PPAR antenna, the accurate polarization measurement could be realized in a wider angle.

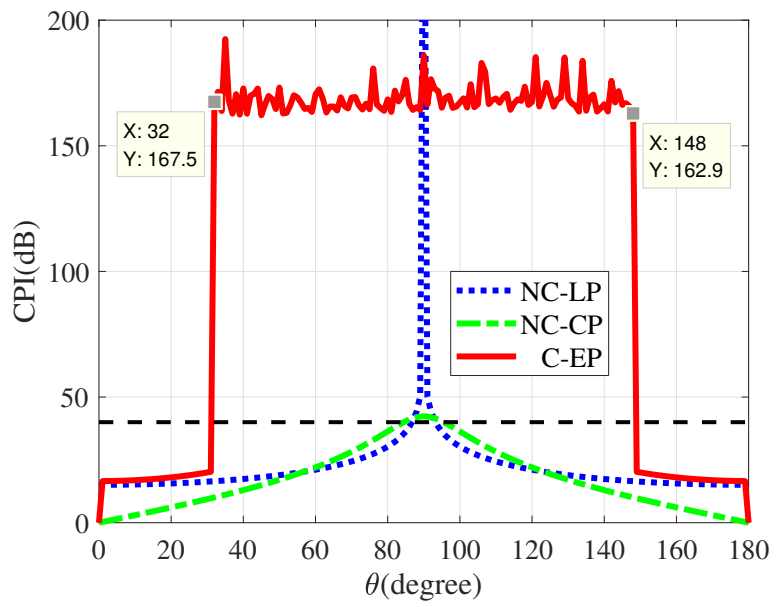

(a)

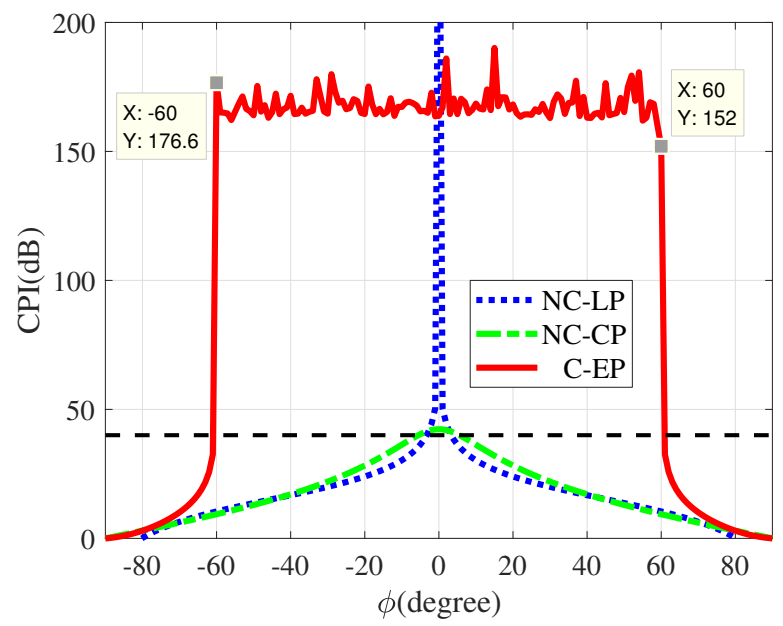

(b)

Fig. 8. Comparison of CPI using different polarization states in a two-dimensional plane. (a) Comparison with different elevations when $\phi=10^{\circ}$. (b) Comparison with different azimuths when $\theta=80^{\circ}$. The results reveal that the C-EP wave has a wider available angle range and greater CPI value.

\section{Parameter Sensitivity Discussion}

The steepest descent method optimizes the objective function $\rho$ iteratively to determine whether to stop the iteration process which is affected by the convergence parameter $\varepsilon$. Hence, $\varepsilon$ influences the iteration times $k$ and the minimum of scalar function $\rho(\varphi, p)$. Each $\rho_{\min }$ corresponds to a pair of polarization parameters including the phase difference $\varphi$ and amplitude ratio $p$.

To explore the impact of $\varepsilon$ on the improved steepest descent method, we conduct the simulations with different $\varepsilon$. Then, the corresponding $\kappa, p, \varphi$, and $\rho_{\min }$ are calculated. The simulation results are displayed in Table I.
TABLE I: The Effect of Convergence Parameter $\varepsilon$

\begin{tabular}{|c|c|c|c|c|c|}
\hline$(\phi, \theta)$ & $\varepsilon$ & $\kappa$ & $p$ & $\varphi$ & $\rho_{\min }(\mathrm{dB})$ \\
\hline \multirow[b]{2}{*}{$\left(0^{\circ}, 90^{\circ}\right)$} & $10^{-2}, 10^{-3}$ & 2 & 1.00 & $90.00^{\circ}$ & -73.81 \\
\hline & $10^{-4} \sim 10^{-6}$ & 3 & 1.00 & $90.00^{\circ}$ & -154.56 \\
\hline \multirow[b]{2}{*}{$\left(10^{\circ}, 80^{\circ}\right)$} & $10^{-2} \sim 10^{-4}$ & 2 & 1.00 & $88.28^{\circ}$ & -89.46 \\
\hline & $10^{-5}, 10^{-6}$ & 3 & 1.00 & $88.28^{\circ}$ & -162.36 \\
\hline \multirow[b]{2}{*}{$\left(20^{\circ}, 70^{\circ}\right)$} & $10^{-2}, 10^{-3}$ & 2 & 1.01 & $83.12^{\circ}$ & -77.99 \\
\hline & $10^{-4} \sim 10^{-6}$ & 3 & 1.01 & $83.12^{\circ}$ & -126.55 \\
\hline \multirow{4}{*}{$\left(30^{\circ}, 60^{\circ}\right)$} & $10^{-2}$ & 2 & 1.04 & $73.95^{\circ}$ & -45.53 \\
\hline & $10^{-3}$ & 3 & 1.04 & $73.95^{\circ}$ & -73.18 \\
\hline & $10^{-4}, 10^{-5}$ & 4 & 1.04 & $73.95^{\circ}$ & -101.24 \\
\hline & $10^{-6}$ & 5 & 1.04 & $73.95^{\circ}$ & -129.14 \\
\hline \multirow{5}{*}{$\left(40^{\circ}, 50^{\circ}\right)$} & $10^{-2}$ & 2 & 1.14 & $61.91^{\circ}$ & -46.56 \\
\hline & $10^{-3}$ & 3 & 1.14 & $61.91^{\circ}$ & -70.74 \\
\hline & $10^{-4}$ & 4 & 1.14 & $61.91^{\circ}$ & -94.91 \\
\hline & $10^{-5}$ & 5 & 1.14 & $61.91^{\circ}$ & -117.96 \\
\hline & $10^{-6}$ & 6 & 1.14 & $61.91^{\circ}$ & -135.53 \\
\hline \multirow{3}{*}{$\left(50^{\circ}, 40^{\circ}\right)$} & $10^{-2}, 10^{-3}$ & 2 & 1.35 & $47.58^{\circ}$ & -62.76 \\
\hline & $10^{-4}$ & 3 & 1.35 & $47.58^{\circ}$ & -92.77 \\
\hline & $10^{-5}, 10^{-6}$ & 4 & 1.35 & $47.58^{\circ}$ & -122.81 \\
\hline \multirow{4}{*}{$\left(60^{\circ}, 30^{\circ}\right)$} & $10^{-2}$ & 1 & 1.32 & $42.42^{\circ}$ & -41.43 \\
\hline & $10^{-3}$ & 2 & 1.32 & $42.42^{\circ}$ & -78.39 \\
\hline & $10^{-4}, 10^{-5}$ & 6 & 1.32 & $42.42^{\circ}$ & -116.07 \\
\hline & $10^{-6}$ & 7 & 1.32 & $42.42^{\circ}$ & -124.74 \\
\hline \multirow[b]{2}{*}{$\left(65^{\circ}, 25^{\circ}\right)$} & $10^{-2}$ & 2 & 1.40 & $32.68^{\circ}$ & -34.57 \\
\hline & $10^{-3}$ & $\geq 10000$ & - & - & - \\
\hline$\left(70^{\circ}, 20^{\circ}\right)$ & $10^{-1}$ & $\geq 10000$ & - & - & - \\
\hline
\end{tabular}

From Table I, it can be observed that $\kappa$ and $\rho_{\min }$ tend to increase and decrease separately with smaller value of $\varepsilon$. The improved steepest descent method could find the minimum of $\rho$ rapidly (greater than $-150 \mathrm{~dB}$ ), and the iteration times does not increase dramatically. Herein, $\varepsilon$ is expected to be as small as possible. However, the smaller value of $\varepsilon$ will result in more computing pressures which will be superimposed in the phased array system with many signal processing channels. Moreover, according to the set requirement of CPI (40 dB), i.e., $\left.\rho_{\text {min }} \leq-40 \mathrm{~dB}\right), \varepsilon=10^{-2}$ is enough even for the wider scanning angle up to $60^{\circ}$. Considering the error of excitation voltage, noise and other errors in the practical issue, $\varepsilon$ would select a smaller value appropriately.

Furthermore, the conclusion discussed above is just applicable for the directions that the angle pointing off the normal of the antenna is not greater than $\left(60^{\circ}, 30^{\circ}\right)$. When the deviation angle is greater than $60^{\circ}, \rho$ is greater than -40 $\mathrm{dB}$. For example, for $(\phi, \theta)=\left(65^{\circ}, 25^{\circ}\right)$, the available $\mathrm{x}$ in Algorithm.1 cannot be found even for $\kappa \geq 10000$ when $\varepsilon=10^{-3}$. For $(\phi, \theta)=\left(70^{\circ}, 20^{\circ}\right)$, the available $\mathrm{x}$ cannot be found even when $\varepsilon=10^{-1}$. The available beam scanning range is limited within $\pm 60^{\circ}$. The slow convergence indicates 


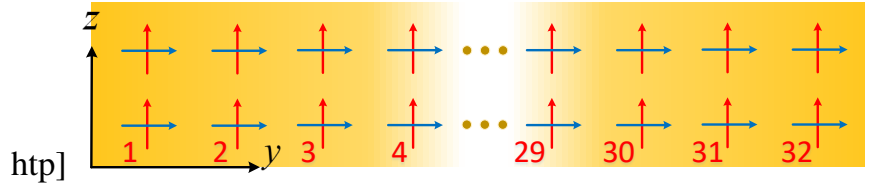

Fig. 9. Constructed phased array with 32 elements.

that the polarimetric performance deteriorates steadily with the gain of beam direction. The reason is that the polarization state configuration is limited by the so-called intrinsic crosspolarization ratio (IXR) [47]. And IXR cannot be eliminated by optimization because it is a property of the antenna [48]. Fortunately, the range is consistent with the common steering range of the actual phased array antenna. Hence, the improved steepest descent method has the efficiency and flexibility for reaching the potential of PPAR.

In summary, the selection of the convergence parameter $\varepsilon$ should be well balanced. When applying this method to the PPAR with other types of elements, the minimum criterion of $\rho$ can be used to select the appropriate convergence parameter $\varepsilon$.

\section{Performance Verification for the Polarimetric PHASED ARRAY}

In this section, the verification is done on the synthesized pattern of PPAR, including the co-polar and cross-polar patterns. In addition, two polarimetric variables are utilized to evaluate the superiority of the $\mathrm{AD}^{2} \mathrm{PSC}$ method on the specific application.

\section{A. Pattern Synthesis}

The PPAR antenna is an array with 32 elements, having 2 rows and 32 columns, as shown in Fig. 9. The first row of elements is used for transmitting and receiving electric field configured by the proposed $\mathrm{AD}^{2} \mathrm{PSC}$ method, while the second row of elements is used only for receiving.

To verify the performance of our proposed method on the PPAR, numerical simulations are realized with the $2 \times 32$ uniformly spaced array. The array antenna is in the yoz plane, and the elements locate along with the $y$ axis. The array antenna has a frequency of $10 \mathrm{GHz}$. The beamforming process is weighted with a $40 \mathrm{~dB}$ Taylor illumination. The synthesized patterns are shown in Fig. 10, which is an illustration based on the radiated polarized wave which is in RHEP state. The situation is similar to that of LHEP wave. A comparison is conducted among the patterns using the NC-LP, NC-CP, and C-EP waves in several scanning directions $(\phi, \theta)=\left(0^{\circ}, 60^{\circ}\right)$, $\left(20^{\circ}, 60^{\circ}\right),\left(40^{\circ}, 60^{\circ}\right)$ and $\left(60^{\circ}, 60^{\circ}\right)$.

As shown in Fig. 10(a), the copolar patterns of NC-LP, NC$\mathrm{CP}$, and C-EP waves, denoted by NC-LP, $\mathrm{NC}_{\mathrm{C}} \mathrm{CP}_{\mathrm{c}}$, and C-EP $\mathrm{c}$ respectively, match basically with each other. However, the cross-polar components, denoted by $\mathrm{NC}-\mathrm{LP}_{\mathrm{x}}, \mathrm{NC}-\mathrm{CP}_{\mathrm{x}}$, and $\mathrm{C}-\mathrm{EP}_{\mathrm{x}}$, are quite different. The cross-polarization $\mathrm{NC}-\mathrm{CP}_{\mathrm{x}}$ is greater than NC-LP $\mathrm{P}_{\mathrm{x}}$ and $\mathrm{NC}-\mathrm{EP}_{\mathrm{x}}$. It is noteworthy that $\mathrm{NC}-\mathrm{LP}_{\mathrm{x}}$ is lower than $\mathrm{NC}-\mathrm{CP}_{\mathrm{x}}$. This is due to that the direction $\left(0^{\circ}, 60^{\circ}\right)$ is on the principle plane of antenna, where the NC-LP wave has greater CPI than NC-CP wave as shown in Fig. 7.
TABLE II: CPI Comparison in $3 \mathrm{~dB}$ Beamwidth of NC-LP, NC-CP, and C-EP States

\begin{tabular}{cccccccccc}
\hline \multirow{2}{*}{$(\phi, \theta)$} & \multicolumn{3}{c}{ NC-LP } & \multicolumn{3}{c}{ NC-CP } & \multicolumn{3}{c}{ C-EP } \\
\cline { 2 - 10 } & Min & Max & Mean & Min & Max & Mean & Min & Max & Mean \\
\hline$\left(0^{\circ}, 60^{\circ}\right)$ & 36.32 & $\mathbf{3 1 9 . 0 9}$ & 61.07 & 22.85 & 22.88 & 22.87 & 36.32 & 174.22 & $\mathbf{5 1 . 4 2}$ \\
\hline$\left(20^{\circ}, 60^{\circ}\right)$ & 14.00 & 15.66 & 14.81 & 19.30 & 20.23 & 19.77 & 34.33 & $\mathbf{1 6 4 . 8 7}$ & $\mathbf{4 9 . 0 7}$ \\
\hline$\left(40^{\circ}, 60^{\circ}\right)$ & 7.54 & 7.70 & 7.62 & 13.88 & 14.03 & 13.95 & 41.45 & $\mathbf{1 5 9 . 1 9}$ & $\mathbf{4 6 . 0 3}$ \\
\hline$\left(60^{\circ}, 60^{\circ}\right)$ & 1.51 & 1.68 & 1.60 & 8.26 & 8.40 & 8.33 & 31.86 & $\mathbf{1 2 1 . 7 7}$ & $\mathbf{4 0 . 3 0}$ \\
\hline
\end{tabular}

With the increasing deviation angle pointing off the normal of the array plane as depicted in Fig. 10(b)-(d), the beam broadens and the cross-polarization levels of three types of polarization wave all raise. For the other three directions, the advantage of C-EP wave is more dominant with respect to $\mathrm{NC}$ LP and NC-CP waves. The C-EP $P_{x}$ is lower than NC-LP ${ }_{x}$ and $\mathrm{NC}-\mathrm{CP}_{\mathrm{x}}$, especially at each boresight location with the sharp concave point. In addition, the cross-polarization component $\mathrm{C}-\mathrm{EP}_{\mathrm{x}}$ still has a lower level in the $3 \mathrm{~dB}$ beamwidth. The specific comparison is listed in Table. II. The CPI decreases with the gain of beam direction pointing away from the antenna normal. The $\mathrm{NC}-\mathrm{LP}_{\mathrm{x}}$ and $\mathrm{NC}-\mathrm{CP}_{\mathrm{x}}$ has relatively more serious deterioration. However, the CPI of configured C-EP wave is greater than $120 \mathrm{~dB}$ even at $(\phi, \theta)=\left(60^{\circ}, 60^{\circ}\right)$, and it has greater CPI which is over $31.86 \mathrm{~dB}$ within $3 \mathrm{~dB}$ beamwidth herein. The mean value which indicates the mean of CPI at all the angle samples within the $3 \mathrm{~dB}$ beamwidth is generally greater than $40 \mathrm{~dB}$.

\section{B. Polarimetric Variables}

The biases of the polarimetric variables $Z_{D R}$ and $L D R$ are displayed in Fig. 11. The elevation angle $\theta=$ $90^{\circ}, 70^{\circ}, 50^{\circ}, 30^{\circ}$, and the azimuth angle $\phi$ varies gradually from $-65^{\circ}$ to $65^{\circ}$ with $10^{\circ}$ interval. If the radiated wave is the NC-LP when $\theta=90^{\circ}$, the absolute value of $\Delta Z_{D R}$ decreases from $14.96 \mathrm{~dB}$ to around $0 \mathrm{~dB}$ when the beam direction approaches the normal of antenna plane as shown in Fig. 11(a1). Then, it increases to $14.96 \mathrm{~dB}$ again once the beam direction points to $\left(65^{\circ}, 90^{\circ}\right)$. The electronically steering deteriorates $\Delta Z_{D R}$, which restricts the available scanning range. Thus, for accurate polarization measurement, the NC-LP wave is suitable just in a limited angle close to the normal direction of the antenna plane. If the radiated wave is NC-CP or C-EP, $\Delta Z_{D R}$ reduces considerably. The bias is around $0 \mathrm{~dB}$ and lower than $0.1 \mathrm{~dB}$ in the whole scanning range which could meet the requirement of accuracy in polarization measurement. Moreover, the polarization measurement may be implemented in the $3 \mathrm{~dB}$ beamwidth of the antenna pattern. $\Delta Z_{D R}$ is assessed when the scattered wave is incident on the half-power $(-3 \mathrm{~dB})$ point. The simulations indicate that the measurement performance at each boresight location is basically equivalent to that in the $3 \mathrm{~dB}$ beamwidth direction.

As shown in Fig.11(b1), if the NC-CP wave is used, the $\triangle L D R$ reduces from $-3.14 \mathrm{~dB}$ to -48.38 and then increases to -4.51 again. The bias index is too great to meet the requirement of accurate polarization measurement, especially 


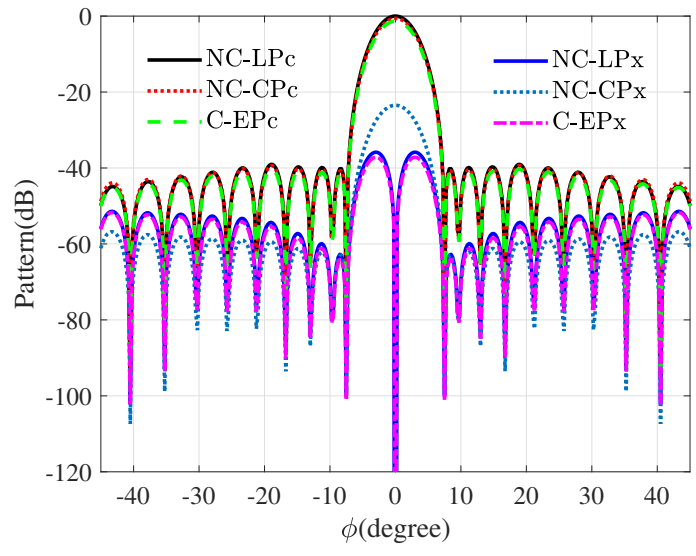

(a)

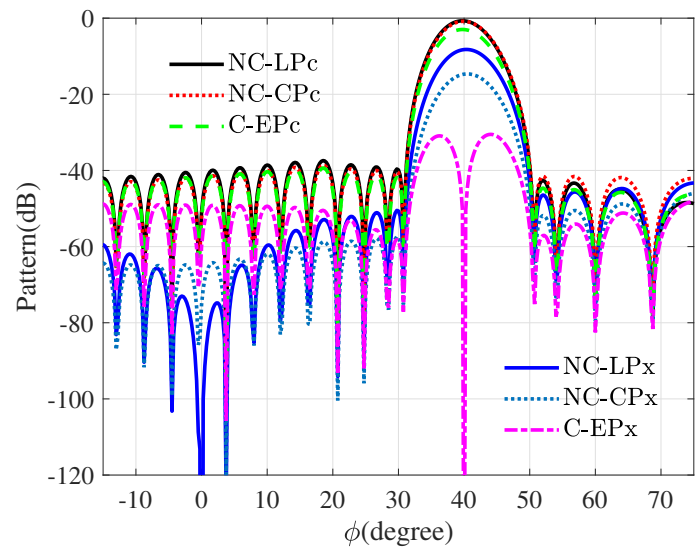

(c)

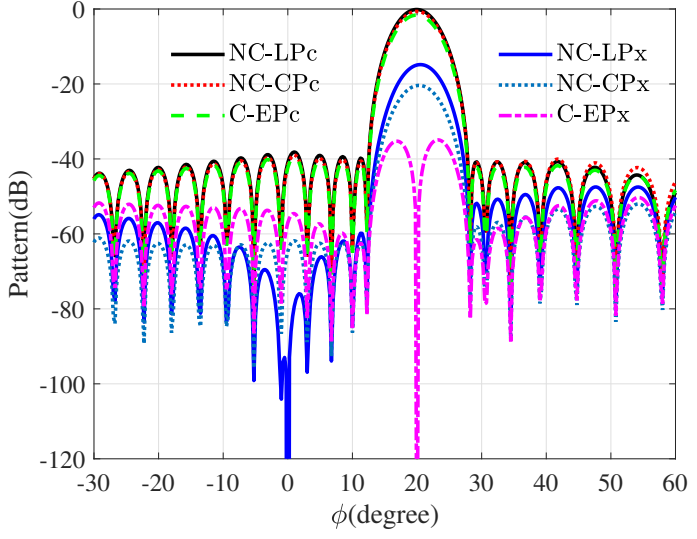

(b)

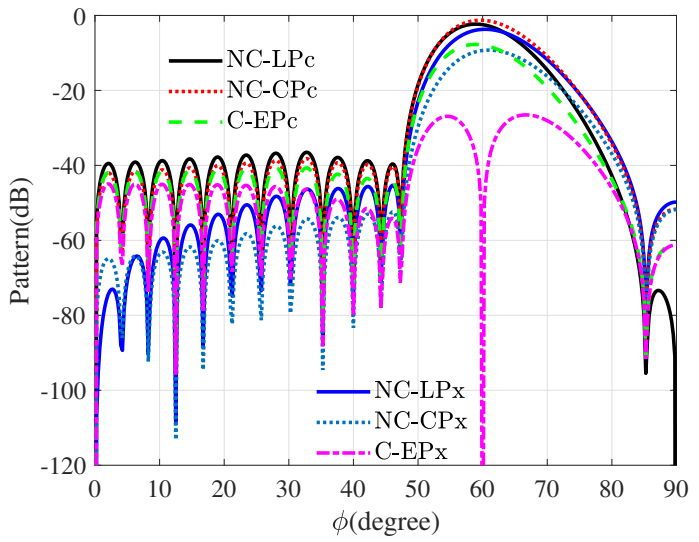

(d)

Fig. 10. The array pattern including the co- and cross-polarization components. (a) $(\phi, \theta)=\left(0^{\circ}, 60^{\circ}\right)$. (b) $(\phi, \theta)=\left(20^{\circ}, 60^{\circ}\right)$. (c) $(\phi, \theta)=\left(40^{\circ}, 60^{\circ}\right)$. (d) $(\phi, \theta)=\left(60^{\circ}, 60^{\circ}\right)$. The results reveal that the cross-polarization of configured C-EP wave is lower than that of NC-LP and NC-CP waves even when the beam direction is up to $60^{\circ}$, thereby heightening the CPI.

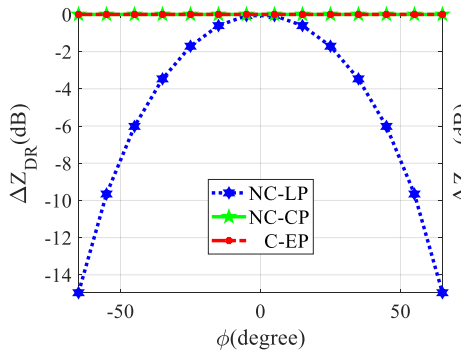

(a1)

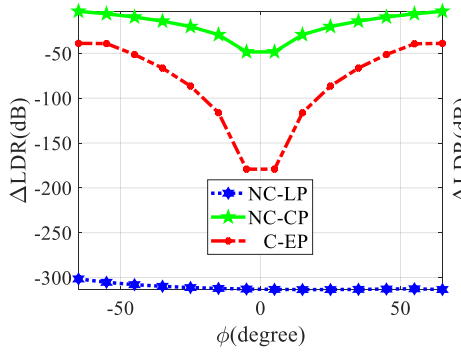

(b1)

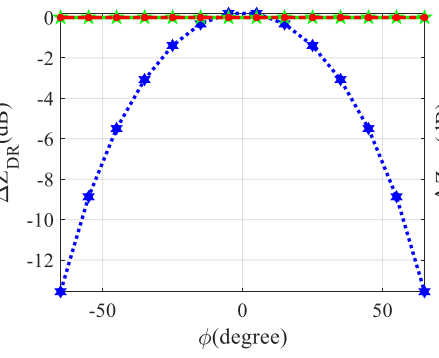

(a2)

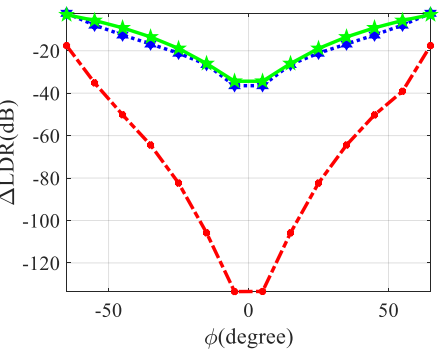

(b2)

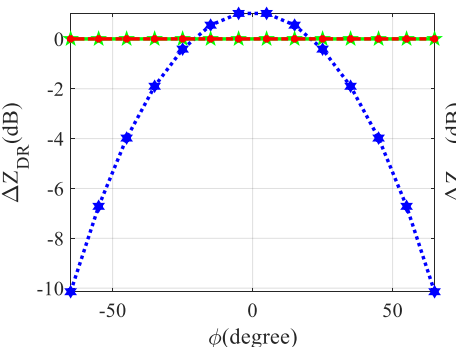

(a3)

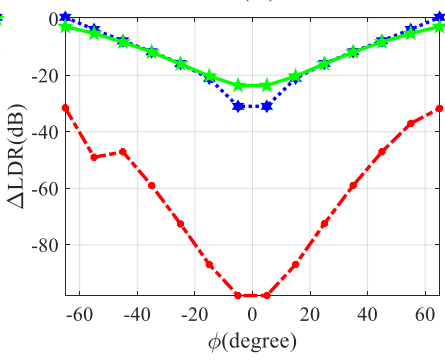

(b3)

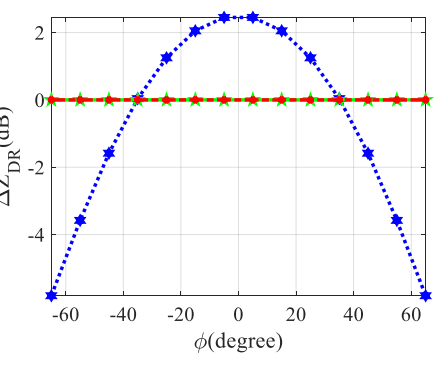

(a4)

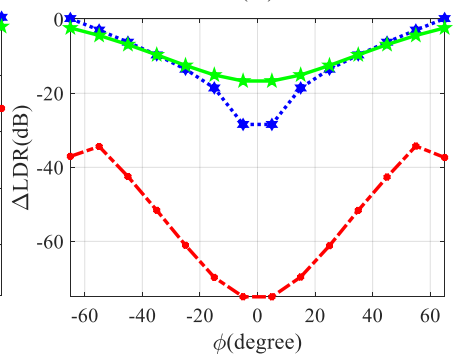

(b4)

Fig. 11. Polarimetric variables versus deviation angle. (a) $\Delta Z_{D R}$ with $\phi$ from $-65^{\circ}$ to $65^{\circ}$ when $\theta=90^{\circ}, 70^{\circ}, 50^{\circ}, 30^{\circ}$. (b) $\triangle L D R$ with $\phi$ from $-65^{\circ}$ to $65^{\circ}$ when $\theta=90^{\circ}, 70^{\circ}, 50^{\circ}, 30^{\circ}$. The results show that the C-EP wave could reduce not only the $\Delta Z_{D R}$ to less than $0.1 \mathrm{~dB}$ but also $\Delta L D R$ to lower than $-40 \mathrm{~dB}$ in a wider angle range, thereby enhancing the polarization measurement accuracy. 
when the beam direction is directed away from the normal plane. It is not the ideal situation for the ATSR and STSR modes. For the NC-LP wave, the $\Delta L D R$ is less than $-300 \mathrm{~dB}$ when $(\phi, \theta)$ varying from $\left(-65^{\circ}, 90^{\circ}\right)$ to $\left(65^{\circ}, 90^{\circ}\right)$. It shows that the cross-polarization is almost negligible in the principle plane when $\theta=90^{\circ}$. However, the performance of NC-LP deteriorates dramatically once the beam direction is pointed off the normal plane as depicted in Fig.11(b2)-(b4). Compared with the NC-LP and NC-CP waves, the polarimetric variable $\triangle L D R$ decreases significantly when the radiated wave is in the C-EP state. As shown in Fig.11(b1), $\triangle L D R$ decreases from a maximum $-38.9 \mathrm{~dB}$ to a minimum $-179.2 \mathrm{~dB}$. Moreover, $\triangle L D R$ increases up to $-38.9 \mathrm{~dB}$ again when the beam deviates the normal gradually. In the overall scanning angle, $\Delta L D R$ is lower than $-38.9 \mathrm{~dB}$. In addtion, the performance does not deteriorate considerably when the beam direction pointing off the broadside as shown in Fig.11(b2)-(b4). Therefore, compared with NC-LP and NC-CP waves, the C-EP wave has a wider available angle range for the accurate polarization measurement.

\section{CONCLUSION AND Discussions}

In this paper, we propose an $\mathrm{AD}^{2} \mathrm{PSC}$ method to contribute to the cross-polarization level reduction in a wider beam scanning range for PPAR. This method is implemented by configuring the excitation of the dual-polarization element for each beam pointing. In contrast to the conventional fixed polarization state, the polarization state is direction-dependent and configured adaptively. The adaptive configuration operation is implemented by the proposed improved steepest descent algorithm while seeking the optimal activated amplitude ratio $p$ and phase difference $\varphi$ of each pair of dual-polarization crossed dipoles. The validity of the $\mathrm{AD}^{2} \mathrm{PSC}$ method is illustrated on the pattern synthesis and polarization measurement application in PPAR. In addition to the pattern synthesis and polarimetric variables assessment, the universal expressions of intrinsic and measured backscatter matrices are deduced under different polarization states.

The performance of the proposed $\mathrm{AD}^{2} \mathrm{PSC}$ method is verified based on numerical simulations and comparisons. For the basic element with two pairs of crossed dipoles, the configured CPI is greater than $150 \mathrm{~dB}$ within the angle range greater than $\pm 58^{\circ}$. The array composed of crossed dipoles has a crosspolarization level below $-120 \mathrm{~dB}$ at each boresight location. In addition, two polarimetric variables including the $Z_{D R}$ and $L D R$ are used to evaluate the measurement accuracy. The biases of $Z_{D R}$ and $L D R$ are less than $0.1 \mathrm{~dB}$ and $-40 \mathrm{~dB}$ separately in a wider scanned azimuth range, which could meet well the polarimetric measurement requirements in both ATSR and STSR modes.

The shortcoming of the proposed method is the architecture cost of the phased array system. More T/R modules are needed for the configuration of the arbitrary polarization state. The extra hardware indicates more signal processing complexity. With the improvement of the signal processing capability, the aforementioned weakness could be relieved. In addition, an alternative technique that uses the element multiplexing method in the antenna design will contribute to reducing the number of $T / R$ modules. The triple-feed patch antenna is a promising candidate scheme [49]. Two of the three feed points are selected for transmitting and receiving. And two feed points are selected only for receiving. One feed point is multiplexed in the triple-feed patch. The polarization state configuration could be tested on the phased array composed of the triplefeed patch antenna elements. The scheme will be considered in future works. Furthermore, the future works will also consider the element design, especially the feeding mechanism, which could be optimized for ease of realizing the polarization state configuration.

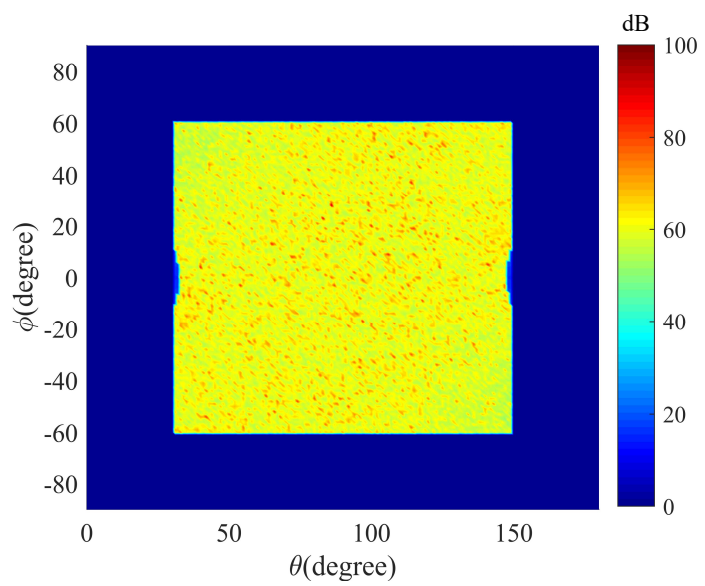

(a)

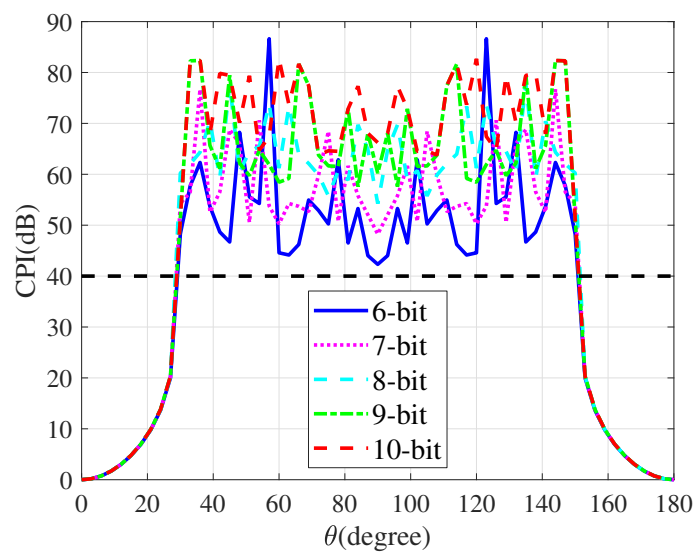

(b)

Fig. 12. The effect of noise or precision on CPI. (a) CPI when $\mathrm{SNR}=20 \mathrm{~dB}$. (b) CPI with the number of quantization bits.

In practice, like the mechanically scanned radar, the polarization measurement performance of a PPAR is also affected by the noise increase or the system error or the excitation precision, which are inevitable in the actual realization. To partially alleviate the problem, we simulate the noise of obtaining the radiation characteristics and assume that the signal to noise (SNR) is $20 \mathrm{~dB}$, the configured CPI is shown as Fig.12(a). The value of CPI is basically greater than $70 \mathrm{~dB}$ within a wider angular sector. The comparisons and simulations above illustrate the feasibility of the proposed method when considering the simulation error.

Furthermore, the array errors such as mutual coupling 
between the elements, imperfection of element radiation characteristics [14], module failure [50], random error of amplitude and phase, and unavoidable quantization of amplitude and phase [51] are also of interest for a PPAR. Among these issues, the number of quantization bits of amplitude and phase would directly affect the precision of the excitation voltage, thereby limiting the effect of polarization state configuration. Through simulating the precision of the excitation amplitude and phase, CPI can still maintain greater than $40 \mathrm{~dB}$ in the expected wide angle range when the number of quantization bits is not less than 6 as shown in Fig.12(b). However, since an experimental platform is not yet available, it is premature to carry out a detailed analysis considering the practical system characteristics.

\section{ACKNOWLEDGMENT}

The authors are very grateful to Taolin Liu for the useful discussions, and we thank the anonymous reviewers, for their valuable advice.

\section{REFERENCES}

[1] M. E. Weber, J. Y. N. Cho, J. S. Herd, J. M. Flavin, W. E. Benner, and G. S. Torok, "The next-generation multimission U.S. surveillance radar network," Bull. Amer. Meteorol. Soc., vol. 88, no. 11, pp. 1739-1751, Nov. 2007.

[2] D. S. Zrnić, J. F. Kimpel, D. E. Forsyth, A. Shapiro, G. Crain, R. Ferek J. Heimmer, W. Benner, T. J. Mcnellis, and R. J. Vogt, "Agile-beam phased array radar for weather observations," Bull. Amer. Meteorol. Soc., vol. 88, no. 11, pp. 1753-1766, Nov. 2007.

[3] G. Zhang, R. J. Doviak, D. S. Zrnić, J. Crain, D. Staiman, and Y. AlRashid, "Phased array radar polarimetry for weather sensing: A theoretical formulation for bias corrections," IEEE Trans. Geosci. Remote Sens., vol. 47, no. 11, pp. 3679-3689, Nov. 2009.

[4] J. S. Herd and M. D. Conway, "The evolution to modern phased array architectures," Proc. IEEE, vol. 104, no. 3, pp. 519-529, Mar. 2016.

[5] J. E. Stailey and K. D. Hondl, "Multifunction phased array radar for aircraft and weather surveillance," Proc. IEEE, vol. 104, no. 3, pp. 649659, Mar. 2016

[6] C. Fulton, J. Salazar, D. Zrnić, D. Mirkovic, I. Ivić, and D. Doviak, "Polarimetrie phased array calibration for large-scale multi-mission radar applications," in 2018 IEEE Radar Conference (RadarConf18), Apr. 2018, pp. $1272-1277$.

[7] G. Zhang, V. Mahale, B. J. Putnam, Y. Qi, Q. Cao, A. Byrd, P. Bukovcic, D. S. Zrnić, J. Gao, M. Xue, Y. Jung, H. Reeves, P. Heinselman, A. Ryzhkov, R. Palmer, P. Zhang, M. Weber, G. Mcfarquhar, B. Moore, and R. Doviak, "Current status and future challenges of weather radar polarimetry: Bridging the gap between radar meteorology/hydrology/engineering and numerical weather prediction," $A d v$ Atmos. Sci., vol. 36, pp. 571-588, Jun. 2019.

[8] C. Fulton, M. Yeary, D. Thompson, J. Lake, and A. Mitchell, "Digital phased arrays: Challenges and opportunities," Proc. IEEE, vol. 104, no. 3 , pp. 487-503, Mar. 2016.

[9] C. Pang, P. Hoogeboom, F. L. Chevalier, H. W. J. Russchenberg, J. Dong, T. Wang, and X. Wang, "Polarimetric bias correction of practical planar scanned antennas for meteorological applications," IEEE Trans. Geosci. Remote Sens., vol. 54, no. 3, pp. 1488-1504, Mar. 2016.

[10] C. Fulton and W. Chappell, "Calibration of panelized polarimetric phased array radar antennas: A case study," in 2010 IEEE International Symposium on Phased Array Systems and Technology, Oct. 2010, pp. 860-867.

[11] L. Lei, G. Zhang, and R. J. Doviak, "Bias correction for polarimetric phased-array radar with idealized aperture and patch antenna elements," IEEE Trans. Geosci. Remote Sens., vol. 51, no. 1, pp. 473-486, Jan. 2013.

[12] C. Pang, J. Dong, T. Wang, and X. Wang, "A polarimetric calibration error model for dual-polarized antenna element patterns," IEEE Antennas Wireless Propag. Lett., vol. 15, pp. 782-785, Aug. 2016.
[13] C. Fulton, J. Herd, S. Karimkashi, G. Zhang, and D. Zrnić, "Dualpolarization challenges in weather radar requirements for multifunction phased array radar," in 2013 IEEE International Symposium on Phased Array Systems and Technology, Oct. 2013, pp. 494-501.

[14] C. Fulton, J. L. Salazar, Y. Zhang, G. Zhang, R. Kelly, J. Meier, M. McCord, D. Schmidt, A. D. Byrd, L. M. Bhowmik, S. Karimkashi, D. S. Zrnić, R. J. Doviak, A. Zahrai, M. Yeary, and R. D. Palmer, "Cylindrical polarimetric phased array radar: Beamforming and calibration for weather applications," IEEE Trans. Geosci. Remote Sens., vol. 55, no. 5, pp. 2827-2841, May 2017.

[15] Z. Wang, C. Pang, Y. Li, and X. Wang, "Bias correction to antenna frequency response for wideband polarimetric phased array radar," Electronics, vol. 8, no. 10, Sep. 2019.

[16] J. L. Salazar, N. Aboserwal, J. D. Díaz, J. A. Ortiz, and C. Fulton, "Edge diffractions impact on the cross polarization performance of active phased array antennas," in 2016 IEEE International Symposium on Phased Array Systems and Technology (PAST), Oct. 2016, pp. 1-5.

[17] Z. Wang, C. Pang, Y. Li, and X. Wang, "Bias correction for polarization measurement in phased array antenna via pattern reconstruction method," IEEE Access, vol. 7, pp. 150006-150012, Oct. 2019.

[18] J. Yin, P. Hoogeboom, C. Unal, H. Russchenberg, F. van der Zwan, and E. Oudejans, "UAV-aided weather radar calibration," IEEE Trans. Geosci. Remote Sens., vol. 57, no. 12, pp. 10362-10375, Dec. 2019.

[19] M. Mirmozafari, G. Zhang, S. Saeedi, and R. J. Doviak, "A dual linear polarization highly isolated crossed dipole antenna for MPAR application," IEEE Antennas Wireless Propag. Lett., vol. 16, pp. 1879-1882, Mar. 2017.

[20] M. Mirmozafari, H. Saeidi-Manesh, and G. Zhang, "Highly isolated crossed dipole antenna with matched copolar beams," Electron. Lett., vol. 54, no. 8, pp. 470-472, Apr. 2018.

[21] J. D. Díaz, J. L. Salazar-Cerreno, J. A. Ortiz, N. A. Aboserwal, R. M. Lebrón, C. Fulton, and R. D. Palmer, "A cross-stacked radiating antenna with enhanced scanning performance for digital beamforming multifunction phased-array radars," IEEE Trans. Antennas Propag., vol. 66, no. 10, pp. 5258-5267, Oct. 2018.

[22] H. Saeidi-Manesh, M. Mirmozafari, and G. Zhang, "Low crosspolarisation high-isolation frequency scanning aperture coupled microstrip patch antenna array with matched dual-polarisation radiation patterns," Electron. Lett., vol. 53, no. 14, pp. 901-902, Jul. 2017.

[23] H. Saeidi-Manesh and G. Zhang, "Dual-polarised hybrid feed microstrip patch antenna for multifunction application," Electron. Lett., vol. 55, no. 12 , pp. 679-681, 2018 .

[24] H. Saeidi-Manesh and G. Zhang, "High-isolation, low cross-polarization, dual-polarization, hybrid feed microstrip patch array antenna for MPAR application," IEEE Trans. Antennas Propag., vol. 66, no. 5, pp. 23262332, May 2018.

[25] H. Saeidi-Manesh and G. Zhang, "Low cross-polarization, high-isolation microstrip patch antenna array for multi-mission applications," IEEE Access, vol. 7, pp. 5026-5033, Dec. 2019.

[26] M. Mirmozafari, G. Zhang, C. Fulton, and R. J. Doviak, "Dualpolarization antennas with high isolation and polarization purity: A review and comparison of cross-coupling mechanisms," IEEE Antennas Propag. Mag., vol. 61, no. 1, pp. 50-63, Feb. 2019.

[27] G. Zhang, R. Doviak, D. Zrnić, R. Palmer, L. Lei, and Y. Al-Rashid, "Polarimetric phased-array radar for weather measurement: A planar or cylindrical configuration?" J. Atmos. Ocean. Technol., vol. 28, no. 1, pp. 63-73, Jan. 2011

[28] T. Sallam, A. B. Abdel-Rahman, M. Alghoniemy, Z. Kawasaki, and T. Ushio, "A neural-network-based beamformer for phased array weather radar," IEEE Trans. Geosci. Remote Sens., vol. 54, no. 9, pp. 5095-5104, Sep. 2016.

[29] B. H. Wang, Y. Guo, Y. L. Wang, and Y. Z. Lin, "Frequency-invariant pattern synthesis of conformal array antenna with low cross-polarisation," IET Microw. Antennas Propag., vol. 2, no. 5, pp. 442-450, Aug. 2008.

[30] J. Xiao and A. Nehorai, "Optimal polarized beampattern synthesis using a vector antenna array," IEEE Trans. Signal Process., vol. 57, no. 2, pp. 576-587, Feb. 2009.

[31] B. Sun, C. Liu, Y. Liu, X. Wu, Y. Li, and X. Wang, "Conformal array pattern synthesis and activated elements selection strategy based on PSOGSA algorithm," Int. J. Antennas Propag., vol. 2015, 2015.

[32] B. Sun, B. Ren, C. Liu, Y. Liu, L. Yongzhen, and X. Wang, "Experimental investigation on the synthesis of scanning beam pattern with antenna selection for conformal array," IET Microw. Antennas Propag., vol. 10, no. 9, pp. 969-975, Jun. 2016.

[33] Y. Liu, J. Bai, K. D. Xu, Z. Xu, F. Han, Q. H. Liu, and Y. Jay Guo, "Linearly polarized shaped power pattern synthesis with sidelobe and 
cross-polarization control by using semidefinite relaxation," IEEE Trans. Antennas Propag., vol. 66, no. 6, pp. 3207-3212, 2018.

[34] D. S. Zrnić, R. Doviak, V. Melnikov, and I. Ivić, "Signal design to suppress coupling in the polarimetric phased array radar," J. Atmos. Ocean. Technol., vol. 31, no. 5, pp. 1063-1077, May 2014.

[35] I. R. Ivić, "Phase code to mitigate the copolar correlation coefficient bias in PPAR weather radar," IEEE Trans. Geosci. Remote Sens., vol. 55 , no. 4, pp. 2144-2166, Apr. 2017.

[36] I. R. Ivić, "An experimental evaluation of phase coding to mitigate the cross-coupling biases in PPAR," in 38th International Conference on Radar Meteorology, Aug. 28-Sep. 1, 2017.

[37] I. R. Ivić, "Options for polarimetrie variable measurements on the MPAR advanced technology demonstrator," in 2018 IEEE Radar Conference (RadarConf18). IEEE, 2018, pp. 0129-0134.

[38] G. Milione, H. Sztul, D. Nolan, and R. Alfano, "Higher-order poincaré sphere, stokes parameters, and the angular momentum of light," Phys. Rev. Lett., vol. 107, no. 5, p. 053601, Jul. 2011.

[39] X. Yi, Y. Liu, X. Ling, X. Zhou, Y. Ke, H. Luo, S. Wen, and D. Fan, "Hybrid-order poincaré sphere," Phys. Rev. A, vol. 91, no. 2, p. 023801, Feb. 2015.

[40] D. Zrnić, R. Doviak, G. Zhang, and A. Ryzhkov, "Bias in differential reflectivity due to cross coupling through the radiation patterns of polarimetric weather radars," J. Atmos. Ocean. Technol., vol. 27, no. 10, pp. 1624-1637, Oct. 2010.

[41] D. S. Zrnić, G. Zhang, and R. J. Doviak, "Bias correction and doppler measurement for polarimetric phased-array radar," IEEE Trans. Geosci. Remote Sens., vol. 49, no. 2, pp. 843-853, Feb. 2011.

[42] T. S. Chu, "Restoring the orthogonality of two polarizations in radio communication systems, i," Bell Syst. Tech. J., vol. 50, no. 9, pp. 30633069, Nov. 1971.

[43] V. Bringi and D. Zrnic, "Role of electromagnetics in polarization radar development from 1970-1995: Personal reflections," 2019. [Online]. Available: https://doi.org/10.20944/preprints201910.0197.v2

[44] V. Bringi and V. Chandrasekar, Polarimetric Doppler weather radar: principles and applications. Cambridge University Press, 2001.

[45] W. M. Boerner, W. L. Yan, A. Q. Xi, and Y. Yamaguchi, "On the basic principles of radar polarimetry: the target characteristic polarization state theory of kennaugh, huynen's polarization fork concept, and its extension to the partially polarized case," Proc. IEEE, vol. 79, no. 10 , pp. 1538-1550, Oct. 1991.

[46] Y. T. Wang and V. Chandrasekar, "Polarization isolation requirements for linear dual-polarization weather radar in simultaneous transmission mode of operation," IEEE Trans. Geosci. Remote Sens., vol. 44, no. 8, pp. 2019-2028, Aug. 2006.

[47] T. D. Carozzi and G. Woan, "A fundamental figure of merit for radio polarimeters," IEEE Trans. Antennas Propag., vol. 59, no. 6, pp. 2058$2065,2011$.

[48] M. V. Ivashina, O. Iupikov, R. Maaskant, W. A. van Cappellen, and T. Oosterloo, "An optimal beamforming strategy for wide-field surveys with phased-array-fed reflector antennas," IEEE Trans. Antennas Propag., vol. 59, no. 6, pp. 1864-1875, 2011.

[49] T. P. Phyoe, E. Nisriyama, and I. Toyoda, "A circularly polarized triplefeed multi-beam array antenna using planar magic- ts," in 2018 IEEE International Symposium on Antennas and Propagation USNC/URSI National Radio Science Meeting, Jul. 2018, pp. 175-176.

[50] M. Levitas, D. A. Horton, and T. C. Cheston, "Practical failure compensation in active phased arrays," IEEE Trans. Antennas Propag., vol. 47, no. 3, pp. 524-535, Mar. 1999.

[51] P. Rocca, G. Oliveri, R. J. Mailloux, and A. Massa, "Unconventional phased array architectures and design methodologies-a review," Proc. IEEE, vol. 104, no. 3, pp. 544-560, Mar. 2016. 